\title{
Nonoscillatory solutions for higher-order nonlinear neutral delay differential equations
}

\author{
Zeqing Liu', Ming Jia², Jeong Sheok Ume ${ }^{3^{*}}$ and Shin Min Kang ${ }^{4}$
}

\section{"Correspondence:}

jsume@changwon.ac.kr

${ }^{3}$ Department of Mathematics,

Changwon National University,

Changwon, 641-773, Korea

Full list of author information is

available at the end of the article

\begin{abstract}
This paper deals with the solvability of the higher-order nonlinear neutral delay differential equation $\frac{d^{n}}{d t^{n}}[x(t)+p(t) x(t-\tau)]+(-1)^{n+1} \sum_{i=1}^{m} q_{i}(t) x\left(\alpha_{i}(t)\right)+(-1)^{n+1} f\left(t_{1}\right.$ $\left.x\left(\beta_{1}(t)\right), \ldots, x\left(\beta_{l}(t)\right)\right)=r(t), t \geq t_{0}$, where $\tau>0, n, m, l \in \mathbb{N}, p, r, q_{i}, \alpha_{i}, \beta_{j} \in C\left(\left[t_{0},+\infty\right), \mathbb{R}\right)$, and $f \in C\left(\left[t_{0},+\infty\right) \times \mathbb{R}^{\prime}, \mathbb{R}\right)$ satisfying $\lim _{t \rightarrow+\infty} \alpha_{i}(t)=\lim _{t \rightarrow+\infty} \beta_{j}(t)=+\infty$, $i \in\{1,2, \ldots, m\}, j \in\{1,2, \ldots, /\}$. With respect to various ranges of the function $p$, we investigate the existence of uncountably many bounded nonoscillatory solutions for the equation. The main tools used in this paper are the Krasnoselskii and Schauder fixed point theorems together with some new techniques. Six nontrivial examples are given to illustrate the superiority of the results presented in this paper.
\end{abstract}

MSC: 39A10; 39A20; 39A22

Keywords: higher-order nonlinear neutral delay differential equation; uncountably many bounded nonoscillatory solutions; Krasnoselskii fixed point theorem; Schauder fixed point theorem

\section{Introduction and preliminaries}

In the past two decades, the oscillation, nonoscillation, and existence of solutions for some kinds of neutral delay differential equations have been extensively studied by many authors. See, for example, [1-16] and the references cited therein.

Recently, Zhang et al. [14] and Öcalan [10] got several existence results of a nonoscillatory solution or positive solution for the first-order neutral delay differential equations

$$
\frac{d}{d t}[x(t)+c(t) x(t-\tau)]+P(t) x(t-\alpha)-Q(t) x(t-\beta)=0, \quad t \geq t_{0}
$$

and

$$
\frac{d}{d t}[x(t)+c(t) x(t-\tau)]+\sum_{i=1}^{m} A_{i}(t) x\left(t-\sigma_{i}\right)-\sum_{i=m+1}^{n} A_{i}(t) x\left(t-\sigma_{i}\right)=0, \quad t \geq t_{0}
$$

where $\tau>0, \alpha, \beta, \sigma_{i} \in \mathbb{R}^{+}, c \in C\left(\left[t_{0},+\infty\right), \mathbb{R}\right)$ and $P, Q, A_{i} \in C\left(\left[t_{0},+\infty\right), \mathbb{R}^{+}\right)$for $i \in$ $\{1,2, \ldots, n\}$. Shen and Debnath [12] obtained some sufficient conditions for the oscillations of (1.1) and Luo and Shen [9] established a few oscillation and nonoscillation criteria for (1.2). Liu and Huang [6] used the coincidence degree theory to get the existence and uniqueness results of a $T$-periodic solution for the first-order neutral functional differen-

\section{然 Springer}

@2014 Liu et al.; licensee Springer. This is an Open Access article distributed under the terms of the Creative Commons Attribution License (http://creativecommons.org/licenses/by/2.0), which permits unrestricted use, distribution, and reproduction in any medium, provided the original work is properly cited. 
tial equation with a deviating argument of the form

$$
\frac{d}{d t}[x(t)+c x(t-\tau)]=g_{1}(t, x(t))+g_{2}(t, x(t-\alpha(t)))+f(t),
$$

where $c, \tau$ are constants, $c \neq \pm 1, f, \alpha \in C(\mathbb{R}, \mathbb{R}), g_{1}, g_{2} \in C\left(\mathbb{R}^{2}, \mathbb{R}\right), f, \alpha$ are $T$-periodic and $g_{1}$, $g_{2}$ are $T$-periodic in the first argument. Using the Banach fixed point theorem, Kulenović and Hadžiomerspahić [4] studied the existence of a nonoscillatory solution for the secondorder neutral delay differential equation with positive and negative coefficients

$$
\frac{d^{2}}{d t^{2}}[x(t)+c x(t-\tau)]+P(t) x(t-\sigma)-Q(t) x(t-\delta)=0, \quad t \geq t_{0},
$$

where $c \in \mathbb{R} \backslash\{ \pm 1\}, \sigma, \delta \in \mathbb{R}^{+}$and $P, Q \in C\left(\left[t_{0},+\infty\right), \mathbb{R}^{+}\right)$. Kong et al. [3] established a complete classification of nonoscillatory solutions for the higher-order neutral differential equation

$$
\frac{d^{n}}{d t^{n}}[x(t)-x(t-\tau)]+f(t) x(t-\alpha)=0, \quad t \geq t_{0},
$$

and gave conditions for each type of nonoscillatory solutions to exist, where $n$ is an odd number, $\tau>0, \alpha \in \mathbb{R}$, and $f \in C\left(\mathbb{R}^{+}, \mathbb{R}^{+}\right)$. Zhou and Zhang [16] extended the results in [5] to higher-order neutral functional differential equation with positive and negative coefficients

$$
\frac{d^{n}}{d t^{n}}[x(t)+c x(t-\tau)]+(-1)^{n+1}[P(t) x(t-\sigma)-Q(t) x(t-\delta)]=0, \quad t \geq t_{0},
$$

where $c \in \mathbb{R} \backslash\{ \pm 1\}, \tau, \sigma, \delta \in \mathbb{R}^{+}$and $P, Q \in C\left(\left[t_{0},+\infty\right), \mathbb{R}^{+}\right)$. Liu et al. [8] investigated the higher-order neutral delay differential equation with positive and negative coefficients

$$
\frac{d^{n}}{d t^{n}}[x(t)+c x(t-\tau)]+(-1)^{n}[P(t) x(f(t))-Q(t) x(g(t))]=0, \quad t \geq t_{0},
$$

where $c \in \mathbb{R} \backslash\{-1\}, \tau \in \mathbb{R}^{+}, P, Q \in C\left(\left[t_{0},+\infty\right), \mathbb{R}^{+}\right), f, g \in C\left(\left[t_{0},+\infty\right), \mathbb{R}\right)$, and $\lim _{t \rightarrow+\infty} f(t)=$ $\lim _{t \rightarrow+\infty} g(t)=+\infty$. Utilizing the Banach fixed point theorem, they obtained the existence of bounded nonoscillatory solutions for (1.7), suggested some algorithms for approximating these bounded nonoscillatory solutions, and discussed the convergence and stability of iteration sequences generated by the algorithms. Parhi [11] discussed the oscillation of solutions for the higher-order neutral delay linear differential equation

$$
\frac{d^{n}}{d t^{n}}[x(t)+c x(t-\tau)]+\sum_{i=1}^{m} q_{i}(t) x\left(t-\alpha_{i}(t)\right)=0, \quad t \geq 0,
$$

where $c \in[0,1), \tau>0, q_{i} \in C\left(\mathbb{R}^{+}, \mathbb{R}\right)$, and $\alpha_{i} \in C\left(\mathbb{R}^{+}, \mathbb{R}^{+}\right)$for $i \in\{1,2, \ldots, m\}$. Li et al. [5] considered the following higher-order neutral delay differential equation with unstable type:

$$
\frac{d^{n}}{d t^{n}}[x(t)+c(t) x(t-\tau)]=q(t)|x(t-\sigma)|^{\alpha-1} x(t-\sigma), \quad t \geq 0,
$$


proved bounded oscillation and nonoscillation criteria and the existence of an unbounded positive solution for (1.9), where $n$ is an even integer, $\alpha \geq 1, \tau>0, \sigma>0$, $c, q \in C\left(\left[t_{0},+\infty\right), \mathbb{R}^{+}\right)$. Zhou and Zhang [15] used the Krasnoselskii and Schauder fixed point theorems to prove the existence of a nonoscillatory solution for the forced higherorder nonlinear neutral functional differential equation

$$
\frac{d^{n}}{d t^{n}}[x(t)+c(t) x(t-\tau)]+\sum_{i=1}^{m} q_{i}(t) f\left(x\left(t-\sigma_{i}\right)\right)=g(t), \quad t \geq t_{0},
$$

where $\tau, \sigma_{i} \in \mathbb{R}^{+}, c, q_{i}, g \in C\left(\left[t_{0},+\infty\right), \mathbb{R}\right)$ for $i \in\{1,2, \ldots, m\}$ and $f \in C(\mathbb{R}, \mathbb{R})$. Liu et al. [7] got the existence of infinitely many nonoscillatory solutions for the $n$ th-order neutral delay differential equation

$$
\frac{d^{n}}{d t^{n}}[x(t)+c x(t-\tau)]+(-1)^{n+1} f\left(t, x\left(t-\sigma_{1}\right), x\left(t-\sigma_{2}\right), \ldots, x\left(t-\sigma_{k}\right)\right)=g(t), \quad t \geq t_{0},
$$

where $c \in \mathbb{R} \backslash\{-1\}, \tau>0, \sigma_{i} \in \mathbb{R}^{+}$for $i \in\{1,2, \ldots, k\}, f \in C\left(\left[t_{0},+\infty\right) \times \mathbb{R}^{k}, \mathbb{R}\right)$, and $g \in$ $C\left(\left[t_{0},+\infty\right), \mathbb{R}^{+}\right)$.

However, to the best of our knowledge, there exist no results for the existence of solutions of the higher-order nonlinear neutral delay differential equation

$$
\begin{aligned}
& \frac{d^{n}}{d t^{n}}[x(t)+p(t) x(t-\tau)]+(-1)^{n+1} \sum_{i=1}^{m} q_{i}(t) x\left(\alpha_{i}(t)\right) \\
& \quad+(-1)^{n+1} f\left(t, x\left(\beta_{1}(t)\right), \ldots, x\left(\beta_{l}(t)\right)\right)=r(t), \quad t \geq t_{0},
\end{aligned}
$$

where $\tau>0, n, m, l \in \mathbb{N}, p, r, q_{i}, \alpha_{i}, \beta_{j} \in C\left(\left[t_{0},+\infty\right), \mathbb{R}\right)$, and $f \in C\left(\left[t_{0},+\infty\right) \times \mathbb{R}^{l}, \mathbb{R}\right)$ satisfying

$$
\lim _{t \rightarrow+\infty} \alpha_{i}(t)=\lim _{t \rightarrow+\infty} \beta_{j}(t)=+\infty, \quad i \in\{1,2, \ldots, m\}, j \in\{1,2, \ldots, l\} .
$$

It is clear that (1.12) includes (1.1)-(1.11) as special cases. The purpose of this paper is to study the solvability of (1.12) under various ranges of the function $p$. Utilizing the Krasnoselskii and Schauder fixed point theorems and some new techniques, we study sufficient conditions of the existence of uncountably many bounded nonoscillatory solutions for (1.12) relative to various ranges of the function $p$. The results presented in this paper extend, improve, and unify the corresponding results in [4] and [13-16]. Six nontrivial examples are also given to illustrate the importance of the results obtained in this paper.

Throughout this paper, we assume that $\mathbb{R}, \mathbb{R}^{+}$and $\mathbb{N}$ denote the sets of all real numbers, nonnegative numbers and positive integers, respectively, and

$$
\gamma=\min \left\{t_{0}-\tau, \inf \left\{\alpha_{i}(t), \beta_{j}(t): t \in\left[t_{0},+\infty\right), i \in\{1,2, \ldots, m\}, j \in\{1,2, \ldots, l\}\right\}\right\} .
$$

Let $C B([\gamma,+\infty), \mathbb{R})$ stand for the Banach space of all continuous and bounded functions on $[\gamma,+\infty)$ with norm $\|x\|=\sup _{t \geq \gamma}|x(t)|$ for all $x \in C B([\gamma,+\infty), \mathbb{R})$ and

$$
A(N, M)=\{x \in C B([\gamma,+\infty), \mathbb{R}): N \leq x(t) \leq M, t \geq \gamma\},
$$


where $M, N \in \mathbb{R}$ with $M>N$. It is easy to see that $A(N, M)$ is a nonempty bounded closed convex subset of $C B([\gamma,+\infty), \mathbb{R})$.

By a solution of (1.12), we mean a function $x \in C([\gamma,+\infty), \mathbb{R})$ for some $T \geq t_{0}$, such that $x(t)+p(t) x(t-\tau)$ is $n$ times continuously differentiable on $[T,+\infty)$ and (1.12) holds for $t \geq T$. As is customary, a solution of (1.12) is said to be oscillatory if it has arbitrarily large zeros and nonoscillatory otherwise.

The following lemmas are well known.

Lamma 1.1 (Krasnoselskii fixed point theorem [1]) Let $X$ be a nonempty bounded closed convex subset of a Banach space $E$ and let $U, S$ be maps of $X$ into $E$ such that $U x+S y \in X$ for every pair $x, y \in X$. If $U$ is a contraction and $S$ is completely continuous, then the equation $U x+S y=x$ has a solution in $X$.

Lamma 1.2 (Schauder fixed point theorem [1]) Let X be a nonempty closed convex subset of a Banach space E. Let $S: X \rightarrow X$ be a continuous mapping such that $S X$ is a relatively compact subset of $X$. Then $S$ has at least one fixed point in $X$.

\section{The existence of uncountably many bounded nonoscillatory solutions}

Now we investigate sufficient conditions for the existence of uncountably many bounded nonoscillatory solutions of (1.12) under various ranges of the function $p$. The proofs of the results presented in this section are based on the Krasnoselskii and Schauder fixed point theorems and a few new and key techniques, one of which is to construct the mappings $U_{L}$ and $S_{L}$ satisfying the conditions in the cited fixed point theorems for each constant $L$, which belongs to certain interval. Let

$$
H(t)=M \sum_{i=1}^{m}\left|q_{i}(t)\right|+|r(t)|+h(t), \quad \forall t \in[T,+\infty) .
$$

Theorem 2.1 Assume that there exist $h \in C\left(\left[t_{0},+\infty\right), \mathbb{R}^{+}\right)$and constants $M, N, p_{0}$, and $c$ satisfying

$$
\begin{aligned}
& \left|f\left(t, u_{1}, \ldots, u_{l}\right)\right| \leq h(t), \quad \forall\left(t, u_{1}, \ldots, u_{l}\right) \in\left[t_{0},+\infty\right) \times[N, M]^{l} ; \\
& \int_{t_{0}}^{+\infty} s^{n-1} \max \left\{\sum_{i=1}^{m}\left|q_{i}(s)\right|,|r(s)|, h(s)\right\} d s<+\infty ; \\
& 0<N<\left(1+p_{0}\right) M, \quad-1<p_{0} \leq p(t) \leq 0, \quad \forall t \geq c \geq t_{0} .
\end{aligned}
$$

Then (1.12) has uncountably many bounded nonoscillatory solutions in $A(N, M)$.

Proof Let $L \in\left(N,\left(1+p_{0}\right) M\right)$. It follows from (2.2) and (2.3) that there exist constants $\theta \in$ $(0,1)$ and $T>\left|t_{0}\right|+\tau+|c|+|\gamma|+n$ sufficiently large satisfying

$$
\theta=\left|p_{0}\right|+\frac{1}{(n-1) !} \int_{T}^{+\infty} s^{n-1} \sum_{i=1}^{m}\left|q_{i}(s)\right| d s
$$

and

$$
\frac{1}{(n-1) !} \int_{T}^{+\infty} s^{n-1} H(s) d s \leq \min \left\{\left(p_{0}+1\right) M-L, L-N\right\} .
$$


Define two mappings $U_{L}$ and $S_{L}: A(N, M) \rightarrow C B([\gamma,+\infty), \mathbb{R})$ by

$$
\begin{aligned}
& \left(U_{L} x\right)(t)= \begin{cases}L-p(t) x(t-\tau)+\frac{1}{(n-1) !} \int_{t}^{+\infty}(s-t)^{n-1} \\
\times\left(\sum_{i=1}^{m} q_{i}(s) x\left(\alpha_{i}(s)\right)+(-1)^{n} r(s)\right) d s, & t \geq T, \\
\left(U_{L} x\right)(T), & \gamma \leq t<T ;\end{cases} \\
& \left(S_{L} x\right)(t)= \begin{cases}\frac{1}{(n-1) !} \int_{t}^{+\infty}(s-t)^{n-1} f\left(s, x\left(\beta_{1}(s)\right), \ldots, x\left(\beta_{l}(s)\right)\right) d s, & t \geq T, \\
\left(S_{L} x\right)(T), & \gamma \leq t<T,\end{cases}
\end{aligned}
$$

for each $x \in A(N, M)$.

First of all we show that

$$
U_{L} x+S_{L} y \in A(N, M), \quad\left\|U_{L} x-U_{L} y\right\| \leq \theta\|x-y\|, \quad \forall x, y \in A(N, M) .
$$

Let $x, y \in A(N, M)$ and $t \geq T$. By (2.3)-(2.6), we get

$$
\begin{aligned}
& \left(U_{L} x\right)(t)+\left(S_{L} y\right)(t) \\
& =L-p(t) x(t-\tau)+\frac{1}{(n-1) !} \int_{t}^{+\infty}(s-t)^{n-1}\left(\sum_{i=1}^{m} q_{i}(s) x\left(\alpha_{i}(s)\right)+(-1)^{n} r(s)\right) d s \\
& +\frac{1}{(n-1) !} \int_{t}^{+\infty}(s-t)^{n-1} f\left(s, y\left(\beta_{1}(s)\right), \ldots, y\left(\beta_{l}(s)\right)\right) d s \\
& \leq L+|p(t)||x(t)| \\
& +\frac{1}{(n-1) !} \int_{t}^{+\infty}(s-t)^{n-1}\left(\sum_{i=1}^{m}\left|q_{i}(s)\right|\left|x\left(\alpha_{i}(s)\right)\right|+|r(s)|\right. \\
& \left.+\left|f\left(s, y\left(\beta_{1}(s)\right), \ldots, y\left(\beta_{l}(s)\right)\right)\right|\right) d s \\
& \leq L-p_{0} M+\frac{1}{(n-1) !} \int_{T}^{+\infty} s^{n-1} H(s) d s \\
& \leq L-p_{0} M+\min \left\{\left(p_{0}+1\right) M-L, L-N\right\} \\
& \leq M \text {, } \\
& \left(U_{L} x\right)(t)+\left(S_{L} y\right)(t) \\
& \geq L-\frac{1}{(n-1) !} \int_{t}^{+\infty}(s-t)^{n-1}\left(\sum_{i=1}^{m}\left|q_{i}(s)\right|\left|x\left(\alpha_{i}(s)\right)\right|+|r(s)|\right. \\
& \left.+\left|f\left(s, y\left(\beta_{1}(s)\right), \ldots, y\left(\beta_{l}(s)\right)\right)\right|\right) d s \\
& \geq L-\frac{1}{(n-1) !} \int_{T}^{+\infty} s^{n-1} H(s) d s \\
& \geq L-\min \left\{\left(p_{0}+1\right) M-L, L-N\right\} \\
& \geq N
\end{aligned}
$$


and

$$
\begin{aligned}
& \left|\left(U_{L} x\right)(t)-\left(U_{L} y\right)(t)\right| \\
& =\mid L-p(t) x(t-\tau)+\frac{1}{(n-1) !} \int_{t}^{+\infty}(s-t)^{n-1}\left(\sum_{i=1}^{m} q_{i}(s) x\left(\alpha_{i}(s)\right)+(-1)^{n} r(s)\right) d s \\
& \quad-L+p(t) y(t-\tau)-\frac{1}{(n-1) !} \int_{t}^{+\infty}(s-t)^{n-1}\left(\sum_{i=1}^{m} q_{i}(s) y\left(\alpha_{i}(s)\right)+(-1)^{n} r(s)\right) d s \mid \\
& \leq-p_{0}\|x-y\|+\frac{1}{(n-1) !} \int_{T}^{+\infty} s^{n-1} \sum_{i=1}^{m}\left|q_{i}(s)\right|\left|x\left(\alpha_{i}(s)\right)-y\left(\alpha_{i}(s)\right)\right| d s \\
& \leq \theta\|x-y\|,
\end{aligned}
$$

which imply (2.7).

Second, we show that $S_{L}$ is continuous in $A(N, M)$ and $S_{L}(A(N, M))$ is relatively compact. Let $\left\{x_{k}\right\}_{k \in \mathbb{N}}$ be an arbitrary sequence in $A(N, M)$ with

$$
\lim _{k \rightarrow \infty} x_{k}=x \in C B([\gamma,+\infty), \mathbb{R}) .
$$

Since $A(N, M)$ is a closed subset of $C B([\gamma,+\infty), \mathbb{R})$, it follows that $x \in A(N, M)$. Put

$$
\begin{aligned}
& G_{k}(s)=\left|f\left(s, x_{k}\left(\beta_{1}(s)\right), \ldots, x_{k}\left(\beta_{l}(s)\right)\right)-f\left(s, x\left(\beta_{1}(s)\right), \ldots, x\left(\beta_{l}(s)\right)\right)\right|, \\
& \forall(s, k) \in[T,+\infty) \times \mathbb{N} .
\end{aligned}
$$

From (2.8) and the continuity of $f$ and $\beta_{j}$ for $j \in\{1,2, \ldots, l\}$ we infer that

$$
\lim _{k \rightarrow \infty} G_{k}(s)=0, \quad \forall s \in[T,+\infty)
$$

which together with (2.6) and the Lebesgue dominated convergence theorem yields for any $t \in[T,+\infty)$

$$
\begin{aligned}
& \left|\left(S_{L} x_{k}\right)(t)-\left(S_{L} x\right)(t)\right| \\
& \quad \leq \frac{1}{(n-1) !} \int_{t}^{+\infty}(s-t)^{n-1}\left|f\left(s, x_{k}\left(\beta_{1}(s)\right), \ldots, x_{k}\left(\beta_{l}(s)\right)\right)-f\left(s, x\left(\beta_{1}(s)\right), \ldots, x\left(\beta_{l}(s)\right)\right)\right| d s \\
& \quad \leq \frac{1}{(n-1) !} \int_{T}^{+\infty} s^{n-1} G_{k}(s) d s \\
& \quad \rightarrow 0 \text { as } k \rightarrow \infty,
\end{aligned}
$$

which gives

$$
\lim _{k \rightarrow \infty}\left\|S_{L} x_{k}-S_{L} x\right\|=\lim _{k \rightarrow \infty} \sup _{t \geq \gamma}\left|\left(S_{L} x_{k}\right)(t)-\left(S_{L} x\right)(t)\right|=\lim _{k \rightarrow \infty} \sup _{t \geq T}\left|\left(S_{L} x_{k}\right)(t)-\left(S_{L} x\right)(t)\right|=0,
$$

which implies that $S_{L}$ is continuous in $A(N, M)$. 
Using (2.1), (2.5), and (2.6), we conclude that for any $x \in A(N, M)$ and $t \geq T$

$$
\begin{aligned}
\left|\left(S_{L} x\right)(t)\right| & =\left|\frac{1}{(n-1) !} \int_{t}^{+\infty}(s-t)^{n-1} f\left(s, x\left(\beta_{1}(s)\right), \ldots, x\left(\beta_{l}(s)\right)\right) d s\right| \\
& \leq \frac{1}{(n-1) !} \int_{T}^{+\infty} s^{n-1} h(s) d s \\
& \leq \min \left\{\left(p_{0}+1\right) M-L, L-N\right\} \\
& \leq M
\end{aligned}
$$

which yields

$$
\left\|S_{L} x\right\| \leq M, \quad \forall x \in A(N, M)
$$

That is, $S_{L}(A(N, M))$ is uniformly bounded in $[\gamma,+\infty)$. In order to prove that $S_{L}(A(N, M))$ is relatively compact, we have to prove that $S_{L}(A(N, M))$ is equicontinuous in $[\gamma,+\infty)$. Let $\varepsilon>0$ be given. Equation (2.2) ensures that there exists $T^{*}>T$ satisfying

$$
\frac{2}{(n-1) !} \int_{T^{*}}^{+\infty} s^{n-1} h(s) d s<\varepsilon
$$

Put

$$
\delta=\min \left\{\frac{\varepsilon}{1+M}, \frac{\varepsilon}{1+\max \left\{h(t): t \in\left[T, T^{*}\right]\right\}}\right\} .
$$

Now we consider the following possible cases:

(i) $t_{2}>t_{1} \geq T^{*}$ with $\left|t_{2}-t_{1}\right|<\delta$. By (2.1), (2.6), and (2.10) we have

$$
\begin{aligned}
& \left|\left(S_{L} x\right)\left(t_{2}\right)-\left(S_{L} x\right)\left(t_{1}\right)\right| \\
& =\frac{1}{(n-1) !} \mid \int_{t_{2}}^{+\infty}\left(s-t_{2}\right)^{n-1} f\left(s, x\left(\beta_{1}(s)\right), \ldots, x\left(\beta_{l}(s)\right)\right) d s \\
& \quad-\int_{t_{1}}^{+\infty}\left(s-t_{1}\right)^{n-1} f\left(s, x\left(\beta_{1}(s)\right), \ldots, x\left(\beta_{l}(s)\right)\right) \mid \\
& \leq \frac{2}{(n-1) !} \int_{T^{*}}^{+\infty} s^{n-1} h(s) d s \\
& <\varepsilon, \quad \forall x \in A(N, M) .
\end{aligned}
$$

(ii) $T \leq t_{1}<t_{2} \leq T^{*}$ with $\left|t_{2}-t_{1}\right|<\delta$ and $n=1$. By means of (2.1), (2.6), and (2.11) we infer that

$$
\begin{aligned}
& \left|\left(S_{L} x\right)\left(t_{2}\right)-\left(S_{L} x\right)\left(t_{1}\right)\right| \\
& \quad=\left|\int_{t_{2}}^{+\infty} f\left(s, x\left(\beta_{1}(s)\right), \ldots, x\left(\beta_{l}(s)\right)\right) d s-\int_{t_{1}}^{+\infty} f\left(s, x\left(\beta_{1}(s)\right), \ldots, x\left(\beta_{l}(s)\right)\right) d s\right| \\
& \quad \leq \int_{t_{1}}^{t_{2}}\left|f\left(s, x\left(\beta_{1}(s)\right), \ldots, x\left(\beta_{l}(s)\right)\right)\right| d s
\end{aligned}
$$




$$
\begin{aligned}
& \leq \int_{t_{1}}^{t_{2}} h(s) d s \\
& <\varepsilon, \quad \forall x \in A(N, M) .
\end{aligned}
$$

(iii) $T \leq t_{1}<t_{2} \leq T^{*}$ with $\left|t_{2}-t_{1}\right|<\delta$ and $n \geq 2$. In light of (2.1), (2.5), and (2.6), we get

$$
\begin{aligned}
\left|\frac{d}{d t}\left(S_{L} x\right)(t)\right| & =\frac{1}{(n-1) !}\left|\frac{d}{d t} \int_{t}^{+\infty}(s-t)^{n-1} f\left(s, x\left(\beta_{1}(s)\right), \ldots, x\left(\beta_{l}(s)\right)\right) d s\right| \\
& =\left|\frac{1}{(n-2) !} \int_{t}^{+\infty}(s-t)^{n-2} f\left(s, x\left(\beta_{1}(s)\right), \ldots, x\left(\beta_{l}(s)\right)\right) d s\right| \\
& \leq \frac{1}{(n-2) !} \int_{T}^{+\infty} s^{n-2} h(s) d s \\
& \leq \frac{1}{(n-1) !} \int_{T}^{+\infty} s^{n-1} h(s) d s \\
& \leq \min \left\{\left(p_{0}+1\right) M-L, L-N\right\} \\
& \leq M, \quad \forall x \in A(N, M), t \in\left[T, T^{*}\right],
\end{aligned}
$$

which together with the mean value theorem and (2.11) yields

$$
\left|\left(S_{L} x\right)\left(t_{2}\right)-\left(S_{L} x\right)\left(t_{1}\right)\right| \leq M\left|t_{1}-t_{2}\right|<\varepsilon, \quad \forall x \in A(N, M) .
$$

(iv) $\gamma \leq t_{1}<t_{2} \leq T$ with $\left|t_{2}-t_{1}\right|<\delta$. Clearly (2.6) means that

$$
\left|\left(S_{L} x\right)\left(t_{2}\right)-\left(S_{L} x\right)\left(t_{1}\right)\right|=0<\varepsilon, \quad \forall x \in A(N, M)
$$

It follows from (2.12)-(2.15) that $S_{L}(A(N, M))$ is equicontinuous in $[\gamma,+\infty)$. Thus Lemma 1.1 means that there exists $x \in A(N, M)$ such that $U_{L} x+S_{L} x=x$. That is,

$$
\begin{aligned}
x(t)= & L-p(t) x(t-\tau)+\frac{1}{(n-1) !} \int_{t}^{+\infty}(s-t)^{n-1}\left(\sum_{i=1}^{m} q_{i}(s) x\left(\alpha_{i}(s)\right)+(-1)^{n} r(s)\right) d s \\
& +\frac{1}{(n-1) !} \int_{t}^{+\infty}(s-t)^{n-1} f\left(s, x\left(\beta_{1}(s)\right), \ldots, x\left(\beta_{l}(s)\right)\right) d s, \quad \forall t \geq T,
\end{aligned}
$$

which implies that

$$
\begin{aligned}
& \frac{d^{n}}{d t^{n}}[x(t)+p(t) x(t-\tau)] \\
& \quad=(-1)^{n}\left(\sum_{i=1}^{m} q_{i}(t) x\left(\alpha_{i}(t)\right)+(-1)^{n} r(t)\right)+(-1)^{n} f\left(t, x\left(\beta_{1}(t)\right), \ldots, x\left(\beta_{l}(t)\right)\right), \quad \forall t \geq T,
\end{aligned}
$$

that is, $x(t)$ is a bounded nonoscillatory solution of $(1.12)$ in $A(N, M)$.

Finally, we show (1.12) has uncountably many bounded nonoscillatory solutions in $A(N, M)$. Let $L_{1}, L_{2} \in\left(N,\left(1+p_{0}\right) M\right)$ with $L_{1} \neq L_{2}$. As in the above proof we can deduce that for each $k \in\{1,2\}$, there exist constants $\theta_{k} \in(0,1), T_{k}>\left|t_{0}\right|+\tau+|c|+|\gamma|+n$, and mappings $U_{L_{k}}, S_{L_{k}}: A(N, M) \rightarrow C B([\gamma,+\infty), \mathbb{R})$ satisfying (2.4)-(2.6), where $\theta, T, L, U_{L}$ and $S_{L}$ are replaced by $\theta_{k}, T_{k}, L_{k}, U_{L_{k}}, S_{L_{k}}$, respectively, and $U_{L_{k}}+S_{L_{k}}$ has a fixed point $z_{k} \in A(N, M)$. 
That is, $z_{1}$ and $z_{2}$ are also bounded nonoscillatory solutions of (1.12) in $A(N, M)$. We now need to show that $z_{1} \neq z_{2}$. In view of (2.2) there exists $T_{3}>\max \left\{T_{1}, T_{2}\right\}$ satisfying

$$
\frac{1}{(n-1) !} \int_{T_{3}}^{+\infty} s^{n-1} h(s) d s<\frac{\left|L_{1}-L_{2}\right|}{4} .
$$

Note that (2.6) means that for $t \geq T_{3}$,

$$
\begin{aligned}
z_{1}(t)= & L_{1}-p(t) z_{1}(t-\tau) \\
& +\frac{1}{(n-1) !} \int_{t}^{+\infty}(s-t)^{n-1}\left(\sum_{i=1}^{m} q_{i}(s) z_{1}\left(\alpha_{i}(s)\right)+(-1)^{n} r(s)\right) d s \\
& +\frac{1}{(n-1) !} \int_{t}^{+\infty}(s-t)^{n-1} f\left(s, z_{1}\left(\beta_{1}(s)\right), \ldots, z_{1}\left(\beta_{l}(s)\right)\right) d s, \\
z_{2}(t)= & L_{2}-p(t) z_{2}(t-\tau) \\
& +\frac{1}{(n-1) !} \int_{t}^{+\infty}(s-t)^{n-1}\left(\sum_{i=1}^{m} q_{i}(s) z_{2}\left(\alpha_{i}(s)\right)+(-1)^{n} r(s)\right) d s \\
& +\frac{1}{(n-1) !} \int_{t}^{+\infty}(s-t)^{n-1} f\left(s, z_{2}\left(\beta_{1}(s)\right), \ldots, z_{2}\left(\beta_{l}(s)\right)\right) d s .
\end{aligned}
$$

It follows from (2.1), (2.4), and (2.17) that for $t \geq T_{3}$

$$
\begin{aligned}
& \mid z_{1}(t)-z_{2}(t) \mid \\
&= \mid L_{1}-L_{2}-p(t)\left(z_{1}(t-\tau)-z_{2}(t-\tau)\right) \\
&+\frac{1}{(n-1) !}\left(\int_{t}^{+\infty}(s-t)^{n-1} \sum_{i=1}^{m} q_{i}(s)\left(z_{1}\left(\alpha_{i}(s)\right)-z_{2}\left(\alpha_{i}(s)\right)\right) d s\right. \\
&+\int_{t}^{+\infty}(s-t)^{n-1}\left(f\left(s, z_{1}\left(\alpha_{1}(s)\right), \ldots, z_{1}\left(\alpha_{l}(s)\right)\right)\right. \\
&\left.\left.-f\left(s, z_{2}\left(\beta_{1}(s)\right), \ldots, z_{2}\left(\beta_{l}(s)\right)\right)\right) d s\right) \mid \\
& \geq\left|L_{1}-L_{2}\right|-\left(-p_{0}+\frac{1}{(n-1) !} \int_{T_{3}}^{+\infty} s^{n-1} \sum_{i=1}^{m}\left|q_{i}(s)\right| d s\right)\left\|z_{1}-z_{2}\right\| \\
& \quad \quad \frac{2}{(n-1) !} \int_{T_{3}}^{+\infty} s^{n-1} h(s) d s \\
& \geq\left|L_{1}-L_{2}\right|-\theta\left\|z_{1}-z_{2}\right\|-\frac{2}{(n-1) !} \int_{T_{3}}^{+\infty} s^{n-1} h(s) d s,
\end{aligned}
$$

which together with (2.16) implies that

$$
\begin{aligned}
\left\|z_{1}-z_{2}\right\| & \geq \frac{1}{1+\theta}\left(\left|L_{1}-L_{2}\right|-\frac{2}{(n-1) !} \int_{T_{3}}^{+\infty} s^{n-1} h(s) d s\right) \\
& >\frac{1}{1+\theta}\left(\left|L_{1}-L_{2}\right|-\frac{\left|L_{1}-L_{2}\right|}{2}\right)
\end{aligned}
$$




$$
\begin{aligned}
& =\frac{\left|L_{1}-L_{2}\right|}{2(1+\theta)} \\
& >0,
\end{aligned}
$$

which yields $z_{1} \neq z_{2}$. This completes the proof.

Theorem 2.2 Assume that there exist $h \in C\left(\left[t_{0},+\infty\right), \mathbb{R}^{+}\right)$and constants $M, N, p_{0}$ and $c$ satisfying (2.1), (2.2), and

$$
0<N<\left(1-p_{0}\right) M, \quad 0 \leq p(t) \leq p_{0}<1, \quad \forall t \geq c \geq t_{0} .
$$

Then (1.12) has uncountably many bounded nonoscillatory solutions in $A(N, M)$.

Proof Let $L \in\left(N,\left(1-p_{0}\right) M\right)$. By (2.2) and (2.18), we choose constants $\theta \in(0,1)$ and $T>$ $\left|t_{0}\right|+\tau+|c|+|\gamma|+n$ satisfying (2.4) and

$$
\frac{1}{(n-1) !} \int_{T}^{+\infty} s^{n-1} H(s) d s \leq \min \left\{M-L, L-p_{0} M-N\right\} .
$$

Define two mappings $U_{L}$ and $S_{L}: A(N, M) \rightarrow C B\left(\left[t_{0},+\infty\right), \mathbb{R}\right)$ by $(2.6)$.

Let $x, y \in A(N, M)$ and $t \geq T$. In terms of (2.6), (2.18), and (2.19), we arrive at

$$
\begin{aligned}
&\left(U_{L} x\right)(t)+\left(S_{L} y\right)(t) \\
&= L-p(t) x(t-\tau)+\frac{1}{(n-1) !} \int_{t}^{+\infty}(s-t)^{n-1}\left(\sum_{i=1}^{m} q_{i}(s) x\left(\alpha_{i}(s)\right)+(-1)^{n} r(s)\right) d s \\
&+\frac{1}{(n-1) !} \int_{t}^{+\infty}(s-t)^{n-1} f\left(s, y\left(\beta_{1}(s)\right), \ldots, y\left(\beta_{l}(s)\right)\right) d s \\
& \leq L \frac{1}{(n-1) !} \int_{t}^{+\infty}(s-t)^{n-1}\left(\sum_{i=1}^{m}\left|q_{i}(s)\right|\left|x\left(\alpha_{i}(s)\right)\right|+|r(s)|\right. \\
&\left.+\left|f\left(s, y\left(\beta_{1}(s)\right), \ldots, y\left(\beta_{l}(s)\right)\right)\right|\right) d s \\
& \leq+\frac{1}{(n-1) !} \int_{T}^{+\infty} s^{n-1} H(s) d s \\
& \leq L+\min \left\{M-L, L-p_{0} M-N\right\} \\
& \leq M \\
&\left(U_{L} x\right)(t)+\left(S_{L} y\right)(t) \\
& \geq L-|p(t)||x(t-\tau)| \\
& \quad \quad \frac{1}{(n-1) !} \int_{t}^{+\infty}(s-t)^{n-1}\left(\sum_{i=1}^{m}\left|q_{i}(s)\right|\left|x\left(\alpha_{i}(s)\right)\right|+|r(s)|\right. \\
& \quad\left.+\left|f\left(s, y\left(\beta_{1}(s)\right), \ldots, y\left(\beta_{l}(s)\right)\right)\right|\right) d s \\
& \geq L-p_{0} M-\frac{1}{(n-1) !} \int_{T}^{+\infty} s^{n-1} H(s) d s
\end{aligned}
$$




$$
\begin{aligned}
& \geq L-p_{0} M-\min \left\{M-L, L-p_{0} M-N\right\} \\
& \geq N
\end{aligned}
$$

which yields $U_{L} x+S_{L} y \in A(N, M)$ for any $x, y \in A(N, M)$. The rest of the proof is similar to that of Theorem 2.1 and is omitted. This completes the proof.

Theorem 2.3 Assume that there exist $h \in C\left(\left[t_{0},+\infty\right), \mathbb{R}^{+}\right)$and constants $M, N, p_{0}, p_{1}$, and c satisfying (2.1), (2.2), and

$$
M>N \max \left\{1, \frac{p_{0}\left(p_{1}^{2}-p_{0}\right)}{p_{1}\left(p_{0}^{2}-p_{1}\right)}\right\}>0, \quad p_{0}^{2}>p_{1} \geq p(t) \geq p_{0}>1, \quad \forall t \geq c \geq t_{0} .
$$

Then (1.12) has uncountably many bounded nonoscillatory solutions in $A(N, M)$.

Proof Let $L \in\left(\frac{p_{1}}{p_{0}} M+p_{1} N, p_{0} M+\frac{p_{0}}{p_{1}} N\right)$. It follows from (2.2) and (2.20) that there exist constants $\theta \in(0,1)$ and $T>\left|t_{0}\right|+\tau+|c|+|\gamma|+n$ satisfying

$$
\theta=\frac{1}{\left|p_{0}\right|}\left(1+\frac{1}{(n-1) !}\right) \int_{T}^{+\infty} s^{n-1} \sum_{i=1}^{m}\left|q_{i}(s)\right| d s
$$

and

$$
\frac{1}{(n-1) !} \int_{T}^{+\infty} s^{n-1} H(s) d s \leq \min \left\{p_{0} M-L+\frac{p_{0}}{p_{1}} N, \frac{p_{0}}{p_{1}} L-M-p_{0} N\right\} .
$$

Define two mappings $U_{L}$ and $S_{L}: A(N, M) \rightarrow C B\left(\left[t_{0},+\infty\right), \mathbb{R}\right)$ by

$$
\begin{aligned}
& \left(U_{L} x\right)(t)= \begin{cases}\frac{L}{p(t+\tau)}-\frac{x(t+\tau)}{p(t+\tau)}+\frac{1}{p(t+\tau)(n-1) !} \int_{t+\tau}^{+\infty}(s-t-\tau)^{n-1} \\
\times\left(\sum_{i=1}^{m} q_{i}(s) x\left(\alpha_{i}(s)\right)+(-1)^{n} r(s)\right) d s, & t \geq T, \\
\left(U_{L} x\right)(T), & \gamma \leq t<T ;\end{cases} \\
& \left(S_{L} x\right)(t)= \begin{cases}\frac{1}{p(t+\tau)(n-1) !} \int_{t+\tau}^{+\infty}(s-t-\tau)^{n-1} f\left(s, x\left(\beta_{1}(s)\right), \ldots, x\left(\beta_{l}(s)\right)\right) d s, & t \geq T, \\
\left(S_{L} x\right)(T), & \gamma \leq t<T .\end{cases}
\end{aligned}
$$

We show that (2.7) holds. In fact, for every $x, y \in A(N, M)$ and $t \geq T$, by (2.1) and (2.20)(2.23), we get

$$
\begin{aligned}
& \left(U_{L} x\right)(t)+\left(S_{L} y\right)(t) \\
& =\frac{L}{p(t+\tau)}-\frac{x(t-\tau)}{p(t+\tau)}+\frac{1}{p(t+\tau)(n-1) !} \\
& \quad \times \int_{t+\tau}^{+\infty}(s-t-\tau)^{n-1}\left(\sum_{i=1}^{m} q_{i}(s) x\left(\alpha_{i}(s)\right)+(-1)^{n} r(s)\right) d s \\
& \quad+\frac{1}{p(t+\tau)(n-1) !} \int_{t+\tau}^{+\infty}(s-t-\tau)^{n-1} f\left(s, y\left(\beta_{1}(s)\right), \ldots, y\left(\beta_{l}(s)\right)\right) d s \\
& \leq \frac{L}{p_{0}}-\frac{N}{p_{1}}+\frac{1}{p_{0}(n-1) !} \int_{T}^{+\infty} s^{n-1} H(s) d s
\end{aligned}
$$




$$
\begin{aligned}
& \quad \leq \frac{L}{p_{0}}-\frac{N}{p_{1}}+\frac{1}{p_{0}} \min \left\{p_{0} M-L+\frac{p_{0}}{p_{1}} N, \frac{p_{0}}{p_{1}} L-M-p_{0} N\right\}=M, \\
& \left(U_{L} x\right)(t)+\left(S_{L} y\right)(t) \\
& \geq \frac{L}{p_{1}}-\frac{M}{p_{0}}-\frac{1}{p_{0}(n-1) !} \int_{T}^{+\infty} s^{n-1} H(s) d s \\
& \geq \frac{L}{p_{1}}-\frac{M}{p_{0}}-\frac{1}{p_{0}} \min \left\{p_{0} M-L+\frac{p_{0}}{p_{1}} N, \frac{p_{0}}{p_{1}} L-M-p_{0} N\right\} \geq N
\end{aligned}
$$

and

$$
\begin{aligned}
\left|\left(U_{L} x\right)(t)-\left(U_{L} y\right)(t)\right| & \mid \frac{L}{p(t+\tau)}-\frac{x(t-\tau)}{p(t+\tau)}+\frac{1}{p(t+\tau)(n-1) !} \\
& \quad \times \int_{t+\tau}^{+\infty}(s-t-\tau)^{n-1}\left(\sum_{i=1}^{m} q_{i}(s) x\left(\alpha_{i}(s)\right)+(-1)^{n} r(s)\right) d s \\
& \quad-\frac{L}{p(t+\tau)}+\frac{y(t-\tau)}{p(t+\tau)}-\frac{1}{p(t+\tau)(n-1) !} \\
& \times \int_{t+\tau}^{+\infty}(s-t-\tau)^{n-1}\left(\sum_{i=1}^{m} q_{i}(s) y\left(\alpha_{i}(s)\right)+(-1)^{n} r(s)\right) d s \mid \\
\leq & \frac{1}{p_{0}}\|x-y\|+\frac{\|x-y\|}{p_{0}(n-1) !} \int_{T}^{+\infty} s^{n-1} \sum_{i=1}^{m}\left|q_{i}(s)\right| d s \\
= & \theta\|x-y\|,
\end{aligned}
$$

which means that we have (2.7).

Next we show that $S_{L}(A(N, M))$ is equicontinuous in $[\gamma,+\infty)$. For any given $\varepsilon>0,(2.2)$ guarantees that (2.10) holds for some sufficiently large $T^{*}>T$. Set

$$
\bar{f}=\max \left\{\left|f\left(t, u_{1}, \ldots, u_{l}\right)\right|: t \in\left[T, T^{*}+\tau\right], u_{j} \in[N, M], j \in\{1,2, \ldots, l\}\right\} .
$$

It follows from the uniform continuity of $p$ in $[N, M]$ that there exists $\delta_{0}>0$ such that

$$
\left|p\left(t_{1}+\tau\right)-p\left(t_{2}+\tau\right)\right|<\frac{\varepsilon}{4(M+N)}
$$

whenever $t_{1}, t_{2} \in\left[T, T^{*}\right]$ with $\left|t_{1}-t_{2}\right|<\delta_{0}$. Put

$$
\delta=\min \left\{\delta_{0}, \frac{\varepsilon}{4 n(M+N)}, \frac{p_{0} \varepsilon}{4(1+\bar{f})\left(T^{*}-T\right)^{n-1}}\right\} .
$$

We have to consider the following possible cases:

(i) $t_{2}>t_{1} \geq T^{*}$ with $\left|t_{2}-t_{1}\right|<\delta$. It follows from (2.1), (2.10), (2.20), (2.23), and (2.26) that

$$
\begin{aligned}
& \left|\left(S_{L} x\right)\left(t_{2}\right)-\left(S_{L} x\right)\left(t_{1}\right)\right| \\
& \quad=\mid \frac{1}{p\left(t_{2}+\tau\right)(n-1) !} \int_{t_{2}+\tau}^{+\infty}\left(s-t_{2}-\tau\right)^{n-1} f\left(s, x\left(\beta_{1}(s)\right), \ldots, x\left(\beta_{l}(s)\right)\right) d s
\end{aligned}
$$




$$
\begin{aligned}
&-\frac{1}{p\left(t_{1}+\tau\right)(n-1) !} \int_{t_{1}+\tau}^{+\infty}\left(s-t_{1}-\tau\right)^{n-1} f\left(s, x\left(\beta_{1}(s)\right), \ldots, x\left(\beta_{l}(s)\right)\right) \mid \\
& \leq \frac{2}{p_{0}(n-1) !} \int_{T^{*}}^{+\infty} s^{n-1} h(s) d s \\
&<\varepsilon, \quad \forall x \in A(N, M) .
\end{aligned}
$$

(ii) $T \leq t_{1}<t_{2} \leq T^{*}$ with $\left|t_{2}-t_{1}\right|<\delta$. For each $s \in\left(t_{2}+\tau,+\infty\right)$, it follows from the mean value theorem that there exists $\xi \in\left(s-t_{2}-\tau, s-t_{1}-\tau\right)$ satisfying

$$
\left(s-t_{2}-\tau\right)^{n-1}-\left(s-t_{1}-\tau\right)^{n-1}=(n-1) \xi^{n-2}\left(t_{1}-t_{2}\right),
$$

which together with (2.1), (2.20), (2.22), and (2.26) yields for each $n \geq 2$

$$
\begin{aligned}
& \frac{1}{p\left(t_{2}+\tau\right)(n-1) !} \int_{t_{2}+\tau}^{+\infty}\left|\left(s-t_{2}-\tau\right)^{n-1}-\left(s-t_{1}-\tau\right)^{n-1}\right|\left|f\left(s, x\left(\beta_{1}(s)\right), \ldots, x\left(\beta_{l}(s)\right)\right)\right| d s \\
& \quad \leq \frac{\left|t_{2}-t_{1}\right|}{p_{0}(n-2) !} \int_{t_{2}+\tau}^{+\infty} \xi^{n-2} h(s) d s \\
& \quad \leq \frac{(n-1)\left|t_{2}-t_{1}\right|}{p_{0}(n-1) !} \int_{T}^{+\infty} s^{n-1} h(s) d s \\
& \quad \leq \frac{n-1}{p_{0}} \cdot \frac{\varepsilon}{4 n(M+N)} \min \left\{p_{0} M-L+\frac{p_{0}}{p_{1}} N, \frac{p_{0}}{p_{1}} L-M-p_{0} N\right\} \\
& \quad<\frac{\varepsilon}{4}, \quad \forall x \in A(N, M),
\end{aligned}
$$

which implies that for each $n \in \mathbb{N}$

$$
\begin{aligned}
& \frac{1}{p\left(t_{2}+\tau\right)(n-1) !} \int_{t_{2}+\tau}^{+\infty}\left|\left(s-t_{2}-\tau\right)^{n-1}-\left(s-t_{1}-\tau\right)^{n-1}\right|\left|f\left(s, x\left(\beta_{1}(s)\right), \ldots, x\left(\beta_{l}(s)\right)\right)\right| d s \\
& \quad<\frac{\varepsilon}{4}, \quad \forall x \in A(N, M) .
\end{aligned}
$$

By means of (2.1), (2.20), (2.22)-(2.26), and (2.28), we get

$$
\begin{aligned}
\left|\left(S_{L} x\right)\left(t_{2}\right)-\left(S_{L} x\right)\left(t_{1}\right)\right| & \\
= & \frac{1}{(n-1) !} \mid \frac{1}{p\left(t_{2}+\tau\right)} \int_{t_{2}+\tau}^{+\infty}\left(s-t_{2}-\tau\right)^{n-1} f\left(s, x\left(\beta_{1}(s)\right), \ldots, x\left(\beta_{l}(s)\right)\right) d s \\
& -\frac{1}{p\left(t_{1}+\tau\right)} \int_{t_{1}+\tau}^{+\infty}\left(s-t_{1}-\tau\right)^{n-1} f\left(s, x\left(\beta_{1}(s)\right), \ldots, x\left(\beta_{l}(s)\right)\right) d s \mid \\
\leq & \frac{1}{(n-1) !}\left(\frac{1}{p\left(t_{2}+\tau\right)} \mid \int_{t_{2}+\tau}^{+\infty}\left(s-t_{2}-\tau\right)^{n-1} f\left(s, x\left(\beta_{1}(s)\right), \ldots, x\left(\beta_{l}(s)\right)\right) d s\right. \\
& -\int_{t_{1}+\tau}^{+\infty}\left(s-t_{1}-\tau\right)^{n-1} f\left(s, x\left(\beta_{1}(s)\right), \ldots, x\left(\beta_{l}(s)\right)\right) d s \mid \\
& \left.+\left|\frac{1}{p\left(t_{2}+\tau\right)}-\frac{1}{p\left(t_{1}+\tau\right)}\right| \int_{t_{1}+\tau}^{+\infty}\left(s-t_{1}-\tau\right)^{n-1}\left|f\left(s, x\left(\beta_{1}(s)\right), \ldots, x\left(\beta_{l}(s)\right)\right)\right| d s\right) \\
\leq & \frac{1}{p\left(t_{2}+\tau\right)(n-1) !}\left(\int_{t_{2}+\tau}^{+\infty}\left|\left(s-t_{2}-\tau\right)^{n-1}-\left(s-t_{1}-\tau\right)^{n-1}\right|\right. \\
\quad & \times\left|f\left(s, x\left(\beta_{1}(s)\right), \ldots, x\left(\beta_{l}(s)\right)\right)\right| d s
\end{aligned}
$$




$$
\begin{aligned}
& \quad+\int_{t_{1}+\tau}^{t_{2}+\tau}\left|\left(s-t_{1}-\tau\right)^{n-1}\right|\left|f\left(s, x\left(\beta_{1}(s)\right), \ldots, x\left(\beta_{l}(s)\right)\right)\right| d s \\
& \left.+\frac{\left|p\left(t_{2}+\tau\right)-p\left(t_{1}+\tau\right)\right|}{p\left(t_{1}+\tau\right)} \int_{t_{1}+\tau}^{+\infty}\left(s-t_{1}-\tau\right)^{n-1}\left|f\left(s, x\left(\beta_{1}(s)\right), \ldots, x\left(\beta_{l}(s)\right)\right)\right| d s\right) \\
& \leq \frac{\varepsilon}{4}+\frac{\left(T^{*}-T\right)^{n-1} \bar{f}}{p_{0}(n-1) !}\left|t_{2}-t_{1}\right|+\frac{\left|p\left(t_{2}+\tau\right)-p\left(t_{1}+\tau\right)\right|}{p_{0}(n-1) !} \int_{T}^{+\infty} s^{n-1} h(s) d s \\
& \leq \frac{\varepsilon}{4}+\frac{\left(T^{*}-T\right)^{n-1} \bar{f}}{p_{0}(n-1) !} \cdot \frac{p_{0} \varepsilon}{4\left(T^{*}-T\right)^{n-1}(1+\bar{f})} \\
& \quad+\frac{\varepsilon}{4(M+N) p_{0}(n-1) !} \min \left\{p_{0} M-L+\frac{p_{0}}{p_{1}} N, \frac{p_{0}}{p_{1}} L-M-p_{0} N\right\} \\
& <\frac{\varepsilon}{4}+\frac{\varepsilon}{4}+\frac{\varepsilon}{4} \\
& <\varepsilon, \quad \forall x \in A(N, M) .
\end{aligned}
$$

(iii) $\gamma \leq t_{1}<t_{2} \leq T$ with $\left|t_{2}-t_{1}\right|<\delta$. Obviously, (2.23) guarantees that

$$
\left|\left(S_{L} x\right)\left(t_{2}\right)-\left(S_{L} x\right)\left(t_{1}\right)\right|=0<\varepsilon, \quad \forall x \in A(N, M) .
$$

Using (2.27), (2.29), and (2.30), we conclude that $S_{L}(A(N, M))$ is equicontinuous in $[\gamma,+\infty)$. As in the proof of Theorem 2.1, we prove similarly that $S_{L}$ is continuous in $A(N, M)$ and $S_{L}(A(N, M))$ is uniformly bounded. It follows that $S_{L}(A(N, M))$ is relatively compact. Consequently, Lemma 1.1 shows that there is $x \in A(N, M)$ such that $U_{L} x+S_{L} x=x$. That is,

$$
\begin{aligned}
x(t)= & \frac{L}{p(t+\tau)}-\frac{x(t+\tau)}{p(t+\tau)} \\
& +\frac{1}{p(t+\tau)(n-1) !} \int_{t+\tau}^{+\infty}(s-t-\tau)^{n-1}\left(\sum_{i=1}^{m} q_{i}(s) x\left(\alpha_{i}(s)\right)+(-1)^{n} r(s)\right) d s \\
& +\frac{1}{p(t+\tau)(n-1) !} \int_{t+\tau}^{+\infty}(s-t-\tau)^{n-1} f\left(s, x\left(\beta_{1}(s)\right), \ldots, x\left(\beta_{l}(s)\right)\right) d s, \quad \forall t \geq T+\tau,
\end{aligned}
$$

which yields

$$
\begin{aligned}
x(t+\tau) & +p(t+\tau) x(t) \\
= & L+\frac{1}{(n-1) !} \int_{t+\tau}^{+\infty}(s-t-\tau)^{n-1}\left(\sum_{i=1}^{m} q_{i}(s) x\left(\alpha_{i}(s)\right)+(-1)^{n} r(s)\right) d s \\
& +\frac{1}{(n-1) !} \int_{t+\tau}^{+\infty}(s-t-\tau)^{n-1} f\left(s, x\left(\beta_{1}(s)\right), \ldots, x\left(\beta_{l}(s)\right)\right) d s, \quad \forall t \geq T+\tau,
\end{aligned}
$$

which implies that

$$
\begin{aligned}
\frac{d^{n}}{d t^{n}}[x(t)+p(t) x(t-\tau)]= & (-1)^{n}\left(\sum_{i=1}^{m} q_{i}(t) x\left(\alpha_{i}(t)\right)+(-1)^{n} r(t)\right) \\
& +(-1)^{n} f\left(t, x\left(\beta_{1}(t)\right), \ldots, x\left(\beta_{l}(t)\right)\right), \quad \forall t \geq T,
\end{aligned}
$$

that is, $x(t)$ is a bounded nonoscillatory solution of $(1.12)$ in $A(N, M)$. 
Finally we show (1.12) has uncountably many bounded nonoscillatory solutions. Let $L_{1}, L_{2} \in\left(\frac{p_{1}}{p_{0}} M+p_{1} N, p_{0} M+\frac{p_{0}}{p_{1}} N\right)$ with $L_{1} \neq L_{2}$. As in the above proof, we infer that for each $k \in\{1,2\}$, there exist constants $\theta_{k} \in(0,1), T_{k}>\left|t_{0}\right|+\tau+|c|+|\gamma|+n$, and mappings $U_{L_{k}}, S_{L_{k}}: A(N, M) \rightarrow C B([\gamma,+\infty), \mathbb{R})$ satisfying (2.21)-(2.23), where $\theta, T, L, U_{L}, S_{L}$ are replaced by $\theta_{k}, T_{k}, L_{k}, U_{L_{k}}, S_{L_{k}}$, respectively, and (1.12) possesses a bounded nonoscillatory solution $z_{k} \in A(N, M)$. In terms of (2.2), we select $T_{3}>\max \left\{T_{1}, T_{2}\right\}$ satisfying

$$
\frac{1}{(n-1) !} \int_{T_{3}}^{+\infty} s^{n-1} h(s) d s<\frac{p_{0}\left|L_{1}-L_{2}\right|}{4 p_{1}}
$$

It follows from (2.21), (2.23), and (2.31) that for $t \geq T_{3}+\tau$

$$
\begin{aligned}
\mid z_{1}(t) & -z_{2}(t) \mid \\
= & \mid \frac{L_{1}-L_{2}}{p(t+\tau)}-\frac{z_{1}(t+\tau)-z_{2}(t+\tau)}{p(t+\tau)} \\
& +\frac{1}{p(t+\tau)(n-1) !}\left(\int_{t+\tau}^{+\infty}(s-t-\tau)^{n-1} \sum_{i=1}^{m} q_{i}(s)\left(z_{1}(\alpha(s))-z_{2}(\alpha(s))\right) d s\right. \\
& +\int_{t+\tau}^{+\infty}(s-t-\tau)^{n-1}\left(f\left(s, z_{1}\left(\beta_{1}(s)\right), \ldots, z_{1}\left(\beta_{l}(s)\right)\right)\right. \\
& \left.\left.-f\left(s, z_{2}\left(\beta_{1}(s)\right), \ldots, z_{2}\left(\beta_{l}(s)\right)\right)\right) d s\right) \mid \\
\geq & \frac{\left|L_{1}-L_{2}\right|}{p_{1}}-\frac{\left\|z_{1}-z_{2}\right\|}{p_{0}}\left(1+\frac{1}{(n-1) !}\right) \int_{T_{3}}^{+\infty} s^{n-1} \sum_{i=1}^{m}\left|q_{i}(s)\right| d s \\
& -\frac{2}{p_{0}(n-1) !} \int_{T_{3}}^{+\infty} s^{n-1} h(s) d s \\
\geq & \frac{\left|L_{1}-L_{2}\right|}{p_{1}}-\theta\left\|z_{1}-z_{2}\right\|-\frac{2}{p_{0}} \cdot \frac{p_{0}\left|L_{1}-L_{2}\right|}{4 p_{1}} \\
= & \frac{\left|L_{1}-L_{2}\right|}{2 p_{1}}-\theta\left\|z_{1}-z_{2}\right\|,
\end{aligned}
$$

which yields

$$
\left\|z_{1}-z_{2}\right\| \geq \frac{\left|L_{1}-L_{2}\right|}{2 p_{1}(1+\theta)}>0
$$

that is, $x_{1} \neq x_{2}$. This completes the proof.

Theorem 2.4 Assume that there exist $h \in C\left(\left[t_{0},+\infty\right), \mathbb{R}^{+}\right)$and constants $M, N, p_{0}, p_{1}$, and c satisfying (2.1), (2.2), and

$$
\begin{aligned}
& M>N>0, \quad \quad p_{0} N\left(1+\frac{1}{p_{1}}\right)>p_{1} M\left(1+\frac{1}{p_{0}}\right), \\
& p_{1} \leq p(t) \leq p_{0}<-1, \quad \forall t \geq c \geq t_{0} .
\end{aligned}
$$

Then (1.12) has uncountably many bounded nonoscillatory solutions in $A(N, M)$. 
Proof Let $L \in\left(p_{1} M\left(1+\frac{1}{p_{0}}\right), p_{0} N\left(1+\frac{1}{p_{1}}\right)\right)$. It follows from (2.2) and (2.32) that there exist constants $\theta \in(0,1)$ and $T>\tau+\left|t_{0}\right|+|c|+|\gamma|$ satisfying (2.21) and

$$
\frac{1}{(n-1) !} \int_{T}^{+\infty} s^{n-1} H(s) d s \leq \min \left\{-M\left(p_{0}+1\right)+\frac{p_{0} L}{p_{1}}, p_{0} N\left(1+\frac{1}{p_{1}}\right)-L\right\} .
$$

Let the mappings $U_{L}$ and $S_{L}: A(N, M) \rightarrow C B\left(\left[t_{0},+\infty\right), \mathbb{R}\right)$ be defined by $(2.23)$.

Note that (2.1), (2.21), (2.23), and (2.33) imply that for each $x, y \in A(N, M)$, and $t \geq T$

$$
\begin{aligned}
\left(U_{L} x\right)(t)+\left(S_{L} y\right)(t) \\
=\frac{L}{p(t+\tau)}-\frac{x(t-\tau)}{p(t+\tau)}+\frac{1}{p(t+\tau)(n-1) !} \\
\quad \times \int_{t+\tau}^{+\infty}(s-t-\tau)^{n-1}\left(\sum_{i=1}^{m} q_{i}(s) x\left(\alpha_{i}(s)\right)+(-1)^{n} r(s)\right) d s \\
\quad+\frac{1}{p(t+\tau)(n-1) !} \int_{t+\tau}^{+\infty}(s-t-\tau)^{n-1} f\left(s, y\left(\beta_{1}(s)\right), \ldots, y\left(\beta_{l}(s)\right)\right) d s \\
\leq \frac{L}{p_{1}}-\frac{M}{p_{0}}-\frac{1}{p_{0}(n-1) !} \int_{T}^{+\infty} s^{n-1} H(s) d s \\
\leq \frac{L}{p_{1}}-\frac{M}{p_{0}}-\frac{1}{p_{0}} \min \left\{-M\left(p_{0}+1\right)+\frac{p_{0} L}{p_{1}}, p_{0} N\left(1+\frac{1}{p_{1}}\right)-L\right\} \\
\leq M
\end{aligned}
$$

and

$$
\begin{aligned}
\left(U_{L} x\right)(t)+\left(S_{L} y\right)(t) \\
=\frac{L}{p(t+\tau)}-\frac{x(t-\tau)}{p(t+\tau)}+\frac{1}{p(t+\tau)(n-1) !} \\
\quad \times \int_{t+\tau}^{+\infty}(s-t-\tau)^{n-1}\left(\sum_{i=1}^{m} q_{i}(s) x\left(\alpha_{i}(s)\right)+(-1)^{n} r(s)\right) d s \\
\quad+\frac{1}{p(t+\tau)(n-1) !} \int_{t+\tau}^{+\infty}(s-t-\tau)^{n-1} f\left(s, y\left(\beta_{1}(s)\right), \ldots, y\left(\beta_{l}(s)\right)\right) d s \\
\geq \frac{L}{p_{0}}-\frac{N}{p_{1}}+\frac{1}{p_{0}(n-1) !} \int_{T}^{+\infty} s^{n-1} H(s) d s \\
\geq \frac{L}{p_{0}}-\frac{N}{p_{1}}+\frac{1}{p_{0}} \min \left\{-M\left(p_{0}+1\right)+\frac{p_{0} L}{p_{1}}, p_{0} N\left(1+\frac{1}{p_{1}}\right)-L\right\} \\
\geq N
\end{aligned}
$$

which yields $U_{L} x+S_{L} y \in A(N, M)$ for any $x, y \in A(N, M)$. The rest of the proof is similar to that of Theorem 2.3 and is omitted. This completes the proof.

Theorem 2.5 Let $n=1$. Assume that there exist $h \in C\left(\left[t_{0},+\infty\right), \mathbb{R}^{+}\right)$and constants $M, N$, and c satisfying (2.1), (2.2), and

$$
0<N<M, \quad p(t) \equiv 1, \quad \forall t \geq c \geq t_{0} .
$$

Then (1.12) has uncountably many bounded nonoscillatory solutions in $A(N, M)$. 
Proof Let $L \in(N, M)$. It follows from (2.2) and (2.34) that there exists a constant $T>\tau+$ $\left|t_{0}\right|+|c|+|\gamma|$ satisfying

$$
\int_{T}^{+\infty} H(s) d s \leq \min \{M-L, L-N\} .
$$

Define a mapping $S_{L}: A(N, M) \rightarrow C B([\gamma,+\infty), \mathbb{R})$ by

$$
\left(S_{L} x\right)(t)= \begin{cases}L+\sum_{a=1}^{\infty} \int_{t+(2 a-1) \tau}^{t+2 a \tau}\left(\sum_{i=1}^{m} q_{i}(s) x\left(\alpha_{i}(s)\right)-r(s)\right. & \\ \left.\quad+f\left(s, x\left(\beta_{1}(s)\right), \ldots, x\left(\beta_{l}(s)\right)\right) d s\right), & t \geq T, \\ \left(S_{L} x\right)(T), & \gamma \leq t<T .\end{cases}
$$

For every $x \in A(N, M)$ and $t \geq T$, by (2.1), (2.35), and (2.36), we deduce that

$$
\begin{aligned}
\left(S_{L} x\right)(t) & =L+\sum_{a=1}^{\infty} \int_{t+(2 a-1) \tau}^{t+2 a \tau}\left(\sum_{i=1}^{m} q_{i}(s) x\left(\alpha_{i}(s)\right)-r(s)+f\left(t, x\left(\beta_{1}(s)\right), \ldots, x\left(\beta_{l}(s)\right)\right)\right) d s \\
& \leq L+\int_{t}^{+\infty} H(s) d s \\
& \leq L+\min \{M-L, L-N\} \\
& \leq M \\
\left(S_{L} x\right)(t) & \geq L-\int_{t}^{+\infty} H(s) d s \\
& \geq L-\min \{M-L, L-N\} \\
& \geq N
\end{aligned}
$$

which yield $S(A(N, M)) \subseteq A(N, M)$ and hence $S(A(N, M))$ is uniformly bounded in $[\gamma,+\infty)$.

Let $\left\{x_{k}\right\}_{k \in \mathbb{N}}$ be a sequence in $A(N, M)$ and $x \in A(N, M)$ satisfying (2.8) and let

$$
\begin{aligned}
G_{k}(s)= & \sum_{i=1}^{m}\left|q_{i}(s)\right|\left|x_{k}\left(\alpha_{i}(s)\right)-x\left(\alpha_{i}(s)\right)\right|+\mid f\left(s, x_{k}\left(\beta_{1}(s)\right), \ldots, x_{k}\left(\beta_{l}(s)\right)\right) \\
& -f\left(s, x\left(\beta_{1}(s)\right), \ldots, x\left(\beta_{l}(s)\right)\right) \mid, \quad \forall(s, k) \in[T,+\infty) \times \mathbb{N} .
\end{aligned}
$$

Using (2.8), (2.37), and the continuity of $f, q_{i}, \alpha_{i}$, and $\beta_{j}$ for $i \in\{1,2, \ldots, m\}$ and $j \in$ $\{1,2, \ldots, l\}$, we obtain $\lim _{t \rightarrow+\infty} G_{k}(s)=0$ for all $s \in[T,+\infty)$. In light of (2.36) and the Lebesgue dominated convergence theorem, we conclude that for any $t \geq T$

$$
\begin{aligned}
& \left|\left(S_{L} x_{k}\right)(t)-\left(S_{L} x\right)(t)\right| \\
& \leq \sum_{a=1}^{\infty} \int_{t+(2 a-1) \tau}^{t+2 a \tau}\left(\sum_{i=1}^{m}\left|q_{i}(s)\right|\left|x_{k}\left(\alpha_{i}(s)\right)-x\left(\alpha_{i}(s)\right)\right|\right. \\
& \left.\quad+\left|f\left(s, x_{k}\left(\beta_{1}(t)\right), \ldots, x_{k}\left(\beta_{l}(t)\right)\right)-f\left(s, x\left(\beta_{1}(t)\right), \ldots, x\left(\beta_{l}(s)\right)\right)\right|\right) d s \\
& \quad \leq \int_{T}^{+\infty} G_{k}(s) d s \rightarrow 0 \quad \text { as } k \rightarrow \infty,
\end{aligned}
$$


which means that

$$
\lim _{k \rightarrow \infty}\left\|S_{L} x_{k}-S_{L} x\right\|=\lim _{k \rightarrow \infty} \sup _{t \geq T}\left|\left(S_{L} x_{k}\right)(t)-\left(S_{L} x\right)(t)\right|=0,
$$

which implies that $S_{L}$ is continuous in $A(N, M)$.

Let $\varepsilon$ be an arbitrary positive number. It follows from (2.2) that there exists $T^{*}>T$ large enough such that

$$
\int_{T^{*}}^{+\infty} H(s) d s<\frac{\varepsilon}{4}
$$

Set

$$
\delta=\frac{\varepsilon}{8 K B} \quad \text { and } \quad B=1+\max \left\{H(s): s \in\left[T, T^{*}+2 K \tau\right]\right\}
$$

where $K \in \mathbb{N}$ satisfies

$$
\begin{aligned}
& \text { either } T+2(K-1) \tau<T^{*} \text { and } T+(2 K-1) \tau \geq T^{*} \text { or } \\
& T+(2 K-1) \tau<T^{*} \text { and } T+2 K \tau \geq T^{*} .
\end{aligned}
$$

We consider the following possible cases:

(i) $t_{2}>t_{1} \geq T^{*}$ with $\left|t_{2}-t_{1}\right|<\delta$. From (2.1), (2.36), and (2.38), we conclude immediately that

$$
\begin{aligned}
& \left|\left(S_{L} x\right)\left(t_{2}\right)-\left(S_{L} x\right)\left(t_{1}\right)\right| \\
& \leq \sum_{a=1}^{\infty} \int_{t_{2}+(2 a-1) \tau}^{t_{2}+2 a \tau}\left(\sum_{i=1}^{m}\left|q_{i}(s)\right|\left|x\left(\alpha_{i}(s)\right)\right|+|r(s)|+\left|f\left(t, x\left(\beta_{1}(s)\right), \ldots, x\left(\beta_{l}(s)\right)\right)\right|\right) d s \\
& \quad+\sum_{a=1}^{\infty} \int_{t_{1}+(2 a-1) \tau}^{t_{1}+2 a \tau}\left(\sum_{i=1}^{m}\left|q_{i}(s)\right|\left|x\left(\alpha_{i}(s)\right)\right|+|r(s)|+\left|f\left(t, x\left(\beta_{1}(s)\right), \ldots, x\left(\beta_{l}(s)\right)\right)\right|\right) d s \\
& \leq 2 \int_{T^{*}}^{+\infty} H(s) d s \\
& <\frac{\varepsilon}{2}, \quad \forall x \in A(N, M) .
\end{aligned}
$$

(ii) $T \leq t_{1}<t_{2} \leq T^{*}$ with $\left|t_{1}-t_{2}\right|<\delta$. In terms of (2.1) and (2.36)-(2.40), we deduce that

$$
\begin{aligned}
& \left|\left(S_{L} x\right)\left(t_{2}\right)-\left(S_{L} x\right)\left(t_{1}\right)\right| \\
& \leq \mid \sum_{a=1}^{K} \int_{t_{2}+(2 a-1) \tau}^{t_{2}+2 a \tau}\left(\sum_{i=1}^{m} q_{i}(s) x\left(\alpha_{i}(s)\right)-r(s)+f\left(t, x\left(\beta_{1}(s)\right), \ldots, x\left(\beta_{l}(s)\right)\right)\right) d s \\
& \quad+\sum_{a=1}^{K} \int_{t_{1}+2 a \tau}^{t_{2}+(2 a-1) \tau}\left(\sum_{i=1}^{m} q_{i}(s) x\left(\alpha_{i}(s)\right)-r(s)+f\left(t, x\left(\beta_{1}(s)\right), \ldots, x\left(\beta_{l}(s)\right)\right)\right) d s \\
& \quad-\sum_{a=1}^{K} \int_{t_{1}+2 a \tau}^{t_{2}+(2 a-1) \tau}\left(\sum_{i=1}^{m} q_{i}(s) x\left(\alpha_{i}(s)\right)-r(s)+f\left(t, x\left(\beta_{1}(s)\right), \ldots, x\left(\beta_{l}(s)\right)\right)\right) d s
\end{aligned}
$$




$$
\begin{aligned}
& \quad-\sum_{a=1}^{K} \int_{t_{1}+(2 a-1) \tau}^{t_{1}+2 a \tau}\left(\sum_{i=1}^{m} q_{i}(s) x\left(\alpha_{i}(s)\right)-r(s)+f\left(t, x\left(\beta_{1}(s)\right), \ldots, x\left(\beta_{l}(s)\right)\right)\right) d s \mid \\
& +\sum_{a=K+1}^{\infty} \int_{t_{2}+(2 a-1) \tau}^{t_{2}+2 a \tau}\left(\sum_{i=1}^{m}\left|q_{i}(s)\right|\left|x\left(\alpha_{i}(s)\right)\right|+|r(s)|+\left|f\left(t, x\left(\beta_{1}(s)\right), \ldots, x\left(\beta_{l}(s)\right)\right)\right|\right) d s \\
& \quad+\sum_{a=K+1}^{\infty} \int_{t_{1}+(2 a-1) \tau}^{t_{1}+2 a \tau}\left(\sum_{i=1}^{m}\left|q_{i}(s)\right|\left|x\left(\alpha_{i}(s)\right)\right|+|r(s)|+\left|f\left(t, x\left(\beta_{1}(s)\right), \ldots, x\left(\beta_{l}(s)\right)\right)\right|\right) d s \\
& \leq \sum_{a=1}^{K} \int_{t_{1}+2 a \tau}^{t_{2}+2 a \tau} H(s) d s+\sum_{a=1}^{K} \int_{t_{1}+(2 a-1) \tau}^{t_{2}+(2 a-1) \tau} H(s) d s+2 \int_{T^{*}}^{+\infty} H(s) d s \\
& \leq 2 K B\left|t_{2}-t_{1}\right|+\frac{\varepsilon}{2} \\
& <\varepsilon, \quad \forall x \in A(N, M) .
\end{aligned}
$$

(iii) $\gamma \leq t_{1}<t_{2} \leq T$ with $\left|t_{1}-t_{2}\right|<\delta$. Equation (2.36) means that

$$
\left|\left(S_{L} x\right)\left(t_{2}\right)-\left(S_{L} x\right)\left(t_{1}\right)\right|=0<\varepsilon, \quad \forall x \in A(N, M) .
$$

It follows from (2.41)-(2.43) that $S_{L}(A(N, M))$ is equicontinuous in $[\gamma,+\infty)$. Thus Lemma 1.2 means that $S_{L}$ has a fixed point $x \in A(N, M)$, that is, for any $t \geq T+\tau$

$$
x(t)=L+\sum_{a=1}^{\infty} \int_{t+(2 a-1) \tau}^{t+2 a \tau}\left(\sum_{i=1}^{m} q_{i}(s) x\left(\alpha_{i}(s)\right)-r(s)+f\left(s, x\left(\beta_{1}(s)\right), \ldots, x\left(\beta_{l}(s)\right)\right)\right) d s
$$

and

$$
x(t-\tau)=L+\sum_{a=1}^{\infty} \int_{t+2(a-1) \tau}^{t+(2 a-1) \tau}\left(\sum_{i=1}^{m} q_{i}(s) x\left(\alpha_{i}(s)\right)-r(s)+f\left(s, x\left(\beta_{1}(s)\right), \ldots, x\left(\beta_{l}(s)\right)\right)\right) d s
$$

which give for any $t \geq T+\tau$

$$
x(t)+x(t-\tau)=2 L+\int_{t}^{+\infty}\left(\sum_{i=1}^{m} q_{i}(s) x\left(\alpha_{i}(s)\right)-r(s)+f\left(s, x\left(\beta_{1}(s)\right), \ldots, x\left(\beta_{l}(s)\right)\right)\right) d s,
$$

which implies that

$$
\frac{d}{d t}[x(t)+x(t-\tau)]+\sum_{i=1}^{m} q_{i}(t) x\left(\alpha_{i}(t)\right)+f\left(t, x\left(\beta_{1}(t)\right), \ldots, x\left(\beta_{l}(t)\right)\right)=r(t), \quad t \geq T+\tau
$$

that is, $x \in A(N, M)$ is a bounded nonoscillatory solution of (1.12). The rest of the proof is similar to that of Theorem 2.1 and is omitted. This completes the proof.

Theorem 2.6 Let $n \in \mathbb{N} \backslash\{1\}$. Assume that there exist $h \in C\left(\left[t_{0},+\infty\right), \mathbb{R}^{+}\right)$and constants $M, N$, and $c$ satisfying (2.1), (2.2), and (2.34). Then (1.12) has uncountably many bounded nonoscillatory solutions in $A(N, M)$. 
Proof Let $L \in(N, M)$. It follows from (2.2) and (2.34) that there exists a constant $T>\tau+$ $\left|t_{0}\right|+|c|+|\gamma|$ satisfying

$$
\frac{1}{(n-1) !} \int_{T}^{+\infty} s^{n-1} H(s) d s \leq \min \{M-L, L-N\}
$$

Define a mapping $S_{L}: A(N, M) \rightarrow C B([\gamma,+\infty), \mathbb{R})$ by

$$
\left(S_{L} x\right)(t)=\left\{\begin{array}{rlrl}
L+ & \frac{1}{(n-2) !} \sum_{a=1}^{\infty} \int_{t+(2 a-1) \tau}^{t+2 a \tau} d u \int_{u}^{+\infty}(s-u)^{n-2} & \\
\quad \times\left(\sum_{i=1}^{m} q_{i}(s) x\left(\alpha_{i}(s)\right)+(-1)^{n} r(s)\right. & \\
\left.\quad+f\left(s, x\left(\beta_{1}(s)\right), \ldots, x\left(\beta_{l}(s)\right)\right)\right) d s, & & t \geq T, \\
\left(S_{L} x\right)(T), & \gamma \leq t<T,
\end{array}\right.
$$

for each $x \in A(N, M)$.

Let $x \in A(N, M)$ and $t \geq T$. By (2.44) and (2.45), we get

$$
\begin{aligned}
\left|\left(S_{L} x\right)(t)-L\right|= & \frac{1}{(n-2) !} \mid \sum_{a=1}^{\infty} \int_{t+(2 a-1) \tau}^{t+2 a \tau} d u \int_{u}^{+\infty}(s-u)^{n-2}\left(\sum_{i=1}^{m} q_{i}(s) x\left(\alpha_{i}(s)\right)\right. \\
& \left.+(-1)^{n} r(s)+f\left(s, x\left(\beta_{1}(s)\right), \ldots, x\left(\beta_{l}(s)\right)\right)\right) d s \mid \\
\leq & \frac{1}{(n-2) !} \int_{t}^{+\infty} d u \int_{u}^{+\infty}(s-u)^{n-2} H(s) d s \\
= & \frac{1}{(n-2) !} \int_{t}^{+\infty} d s \int_{t}^{s}(s-u)^{n-2} H(s) d u \\
= & \frac{1}{(n-1) !} \int_{t}^{+\infty}(s-t)^{n-1} H(s) d s \\
\leq & \min \{M-L, L-N\},
\end{aligned}
$$

which gives that $S(A(N, M)) \subseteq A(N, M)$ and $S(A(N, M))$ is uniformly bounded in $[\gamma,+\infty)$.

Let $\left\{x_{k}\right\}_{k \in \mathbb{N}} \subset A(N, M)$ satisfy (2.8) for some $x \in A(N, M)$ and $\left\{G_{k}\right\}_{k \in \mathbb{N}}$ be defined by (2.37). Using (2.8), (2.37), (2.45), the continuity of $f$ and $\beta_{j}$ for $j \in\{1,2, \ldots, l\}$, and the Lebesgue dominated convergence theorem, we conclude that for any $t \geq T$

$$
\begin{aligned}
\left|\left(S_{L} x_{k}\right)(t)-\left(S_{L} x\right)(t)\right| & \\
\leq & \frac{1}{(n-2) !} \sum_{a=1}^{\infty} \int_{t+(2 a-1) \tau}^{t+2 a \tau} d u \int_{u}^{+\infty}(s-u)^{n-2}\left(\sum_{i=1}^{m}\left|q_{i}(s)\right|\left|x_{k}\left(\alpha_{i}(s)\right)-x\left(\alpha_{i}(s)\right)\right|\right. \\
& \left.+\left|f\left(s, x_{k}\left(\beta_{1}(t)\right), \ldots, x_{k}\left(\beta_{l}(t)\right)\right)-f\left(s, x\left(\beta_{1}(t)\right), \ldots, x\left(\beta_{l}(s)\right)\right)\right|\right) d s \\
\leq & \frac{1}{(n-2) !} \int_{t}^{+\infty} d u \int_{u}^{+\infty}(s-u)^{n-2} G_{k}(s) d s \\
\leq & \frac{1}{(n-1) !} \int_{T}^{+\infty}(s-T)^{n-1} G_{k}(s) d s \\
\rightarrow & 0 \quad \text { as } k \rightarrow \infty,
\end{aligned}
$$


which yields

$$
\lim _{k \rightarrow \infty}\left\|S_{L} x_{k}-S_{L} x\right\|=\lim _{k \rightarrow \infty} \sup _{t \geq T}\left|\left(S_{L} x_{k}\right)(t)-\left(S_{L} x\right)(t)\right|=0,
$$

that is, $S_{L}$ is continuous in $A(N, M)$.

Next we show that $S_{L}(A(N, M))$ is equicontinuous in $[\gamma,+\infty)$. Let $\varepsilon>0$. Equation (2.34) ensures that there exists $T^{*}>T$ large enough satisfying

$$
\frac{1}{(n-1) !} \int_{T^{*}}^{+\infty} s^{n-1} H(s) d s<\frac{\varepsilon}{16} .
$$

Let $B$ and $K$ be defined by (2.39) and (2.40), respectively. Put

$$
\delta=\frac{\varepsilon}{4\left[n K \min \{M-L, L-N\}+B\left(T^{*}+2 K \tau\right)^{n-1}\right]} .
$$

Now we have to consider the following possible cases:

(i) $t_{2}>t_{1} \geq T^{*}$ with $\left|t_{1}-t_{2}\right|<\delta$. In terms of (2.45)-(2.47), we know that

$$
\begin{aligned}
\left|\left(S_{L} x\right)\left(t_{1}\right)-\left(S_{L} x\right)\left(t_{2}\right)\right| & \\
= & \frac{1}{(n-2) !} \mid \sum_{a=1}^{\infty} \int_{t_{1}+(2 a-1) \tau}^{t_{1}+2 a \tau} d u \int_{u}^{+\infty}(s-u)^{n-2}\left(\sum_{i=1}^{m} q_{i}(s) x\left(\alpha_{i}(s)\right)+(-1)^{n} r(s)\right. \\
& \left.+f\left(s, x\left(\beta_{1}(s)\right), \ldots, x\left(\beta_{l}(s)\right)\right)\right) d s \\
& \quad-\sum_{a=1}^{\infty} \int_{t_{2}+(2 a-1) \tau}^{t_{2}+2 a \tau} d u \int_{u}^{+\infty}(s-u)^{n-2}\left(\sum_{i=1}^{m} q_{i}(s) x\left(\alpha_{i}(s)\right)+(-1)^{n} r(s)\right. \\
& \left.+f\left(s, x\left(\beta_{1}(s)\right), \ldots, x\left(\beta_{l}(s)\right)\right)\right) d s \mid \\
\leq & \frac{1}{(n-2) !}\left(\int_{t_{1}}^{+\infty} d u \int_{u}^{+\infty}(s-u)^{n-2} H(s) d s+\int_{t_{2}}^{+\infty} d u \int_{u}^{+\infty}(s-u)^{n-2} H(s) d s\right) \\
\leq & \frac{2}{(n-1) !} \int_{T^{*}}^{+\infty} s^{n-1} H(s) d s \\
< & \varepsilon, \quad \forall x \in A(N, M) .
\end{aligned}
$$

(ii) $T \leq t_{1}<t_{2} \leq T^{*}$ with $\left|t_{1}-t_{2}\right|<\delta$. By means of (2.39), (2.40), (2.44)-(2.47), and the mean value theorem, we conclude that

$$
\begin{aligned}
& \left|\left(S_{L} x\right)\left(t_{1}\right)-\left(S_{L} x\right)\left(t_{2}\right)\right| \\
& =\frac{1}{(n-2) !} \mid \sum_{a=1}^{K} \int_{t_{1}+(2 a-1) \tau}^{t_{1}+2 a \tau} d u \int_{u}^{+\infty}(s-u)^{n-2}\left(\sum_{i=1}^{m} q_{i}(s) x\left(\alpha_{i}(s)\right)+(-1)^{n} r(s)\right. \\
& \left.\quad+f\left(s, x\left(\beta_{1}(s)\right), \ldots, x\left(\beta_{l}(s)\right)\right)\right) d s \\
& \quad-\sum_{a=1}^{K} \int_{t_{1}+(2 a-1) \tau}^{t_{2}+2 a \tau} d u \int_{u}^{+\infty}(s-u)^{n-2}\left(\sum_{i=1}^{m} q_{i}(s) x\left(\alpha_{i}(s)\right)+(-1)^{n} r(s)\right.
\end{aligned}
$$




$$
\begin{aligned}
& \left.+f\left(s, x\left(\beta_{1}(s)\right), \ldots, x\left(\beta_{l}(s)\right)\right)\right) d s \\
& +\sum_{a=1}^{K} \int_{t_{1}+(2 a-1) \tau}^{t_{2}+2 a \tau} d u \int_{u}^{+\infty}(s-u)^{n-2}\left(\sum_{i=1}^{m} q_{i}(s) x\left(\alpha_{i}(s)\right)+(-1)^{n} r(s)\right. \\
& \left.+f\left(s, x\left(\beta_{1}(s)\right), \ldots, x\left(\beta_{l}(s)\right)\right)\right) d s \\
& -\sum_{a=1}^{K} \int_{t_{2}+(2 a-1) \tau}^{t_{2}+2 a \tau} d u \int_{u}^{+\infty}(s-u)^{n-2}\left(\sum_{i=1}^{m} q_{i}(s) x\left(\alpha_{i}(s)\right)+(-1)^{n} r(s)\right. \\
& \left.+f\left(s, x\left(\beta_{1}(s)\right), \ldots, x\left(\beta_{l}(s)\right)\right)\right) d s \\
& +\sum_{a=K+1}^{\infty} \int_{t_{1}+(2 a-1) \tau}^{t_{1}+2 a \tau} d u \int_{u}^{+\infty}(s-u)^{n-2}\left(\sum_{i=1}^{m} q_{i}(s) x\left(\alpha_{i}(s)\right)+(-1)^{n} r(s)\right. \\
& \left.+f\left(s, x\left(\beta_{1}(s)\right), \ldots, x\left(\beta_{l}(s)\right)\right)\right) d s \\
& -\sum_{a=K+1}^{\infty} \int_{t_{2}+(2 a-1) \tau}^{t_{2}+2 a \tau} d u \int_{u}^{+\infty}(s-u)^{n-2}\left(\sum_{i=1}^{m} q_{i}(s) x\left(\alpha_{i}(s)\right)+(-1)^{n} r(s)\right. \\
& \left.+f\left(s, x\left(\beta_{1}(s)\right), \ldots, x\left(\beta_{l}(s)\right)\right)\right) d s \\
& \leq \frac{1}{(n-2) !}\left[\sum _ { a = 1 } ^ { K } \left(\int_{t_{1}+2 a \tau}^{t_{2}+2 a \tau} d u \int_{u}^{+\infty}(s-u)^{n-2} H(s) d s\right.\right. \\
& \left.+\int_{t_{1}+(2 a-1) \tau}^{t_{2}+(2 a-1) \tau} d u \int_{u}^{+\infty}(s-u)^{n-2} H(s) d s\right) \\
& +\int_{t_{1}+(2 K+1) \tau}^{+\infty} d u \int_{u}^{+\infty}(s-u)^{n-2} H(s) d s \\
& \left.+\int_{t_{2}+(2 K+1) \tau}^{+\infty} d u \int_{u}^{+\infty}(s-u)^{n-2} H(s) d s\right] \\
& \leq \frac{1}{(n-1) !}\left[\sum _ { a = 1 } ^ { K } \left(\int_{t_{2}+2 a \tau}^{+\infty}\left[\left(s-t_{1}-2 a \tau\right)^{n-1}-\left(s-t_{2}-2 a \tau\right)^{n-1}\right] H(s) d s\right.\right. \\
& +\int_{t_{1}+2 a \tau}^{t_{2}+2 a \tau}\left(s-t_{1}-2 a \tau\right)^{n-1} H(s) d s \\
& +\int_{t_{2}+(2 a-1) \tau}^{+\infty}\left[\left(s-t_{1}-(2 a-1) \tau\right)^{n-1}-\left(s-t_{2}-(2 a-1) \tau\right)^{n-1}\right] H(s) d s \\
& \left.+\int_{t_{1}+(2 a-1) \tau}^{t_{2}+(2 a-1) \tau}\left(s-t_{1}-(2 a-1) \tau\right)^{n-1} H(s) d s\right) \\
& +\int_{t_{1}+(2 K+1) \tau}^{+\infty}\left(s-t_{1}-(2 K+1) \tau\right)^{n-1} H(s) d s \\
& \left.+\int_{t_{2}+(2 K+1) \tau}^{+\infty}\left(s-t_{2}-(2 K+1) \tau\right)^{n-1} H(s) d s\right]
\end{aligned}
$$




$$
\begin{aligned}
\leq & \frac{2}{(n-1) !}\left(K(n-1)\left|t_{1}-t_{2}\right| \int_{T^{*}}^{+\infty} s^{n-2} H(s) d s\right. \\
& \left.+B\left(T^{*}+2 K \tau\right)^{n-1}\left|t_{1}-t_{2}\right|+\int_{T^{*}}^{+\infty} s^{n-1} H(s) d s\right) \\
\leq & 2\left[K(n-1) \min \{M-L, L-N\}+B\left(T^{*}+2 K \tau\right)^{n-1}\right]\left|t_{1}-t_{2}\right|+\frac{\varepsilon}{8} \\
< & \varepsilon, \quad \forall x \in A(N, M) .
\end{aligned}
$$

(iii) $\gamma \leq t_{1}<t_{2} \leq T$ with $\left|t_{1}-t_{2}\right|<\delta$. It is easy to see that

$$
\left|\left(S_{L} x\right)\left(t_{2}\right)-\left(S_{L} x\right)\left(t_{1}\right)\right|=0<\varepsilon .
$$

It follows from (2.48)-(2.50) that $S_{L}(A(N, M))$ is equicontinuous in $[\gamma,+\infty)$. Consequently $S_{L}(A(N, M))$ is relatively compact. Thus Lemma 1.2 ensures that $S_{L}$ possesses a fixed point $x \in A(N, M)$, that is,

$$
\begin{aligned}
x(t)= & L+\frac{1}{(n-2) !} \sum_{a=1}^{\infty} \int_{t+(2 a-1) \tau}^{t+2 a \tau} d u \int_{u}^{+\infty}(s-u)^{n-2}\left(\sum_{i=1}^{m} q_{i}(s) x\left(\alpha_{i}(s)\right)\right. \\
& \left.+(-1)^{n} r(s)+f\left(s, x\left(\beta_{1}(s)\right), \ldots, x\left(\beta_{l}(s)\right)\right)\right) d s, \quad t \geq T+\tau
\end{aligned}
$$

and

$$
\begin{aligned}
x(t-\tau)= & L+\frac{1}{(n-2) !} \sum_{a=1}^{\infty} \int_{t+2(a-1) \tau}^{t+(2 a-1) \tau} d u \int_{u}^{+\infty}(s-u)^{n-2}\left(\sum_{i=1}^{m} q_{i}(s) x\left(\alpha_{i}(s)\right)\right. \\
& \left.+(-1)^{n} r(s)+f\left(s, x\left(\beta_{1}(s)\right), \ldots, x\left(\beta_{l}(s)\right)\right)\right) d s, \quad t \geq T,
\end{aligned}
$$

which imply that

$$
\begin{aligned}
x(t)+ & x(t-\tau) \\
= & 2 L+\frac{1}{(n-2) !} \int_{t}^{+\infty} d u \int_{u}^{+\infty}(s-u)^{n-2}\left(\sum_{i=1}^{m} q_{i}(s) x\left(\alpha_{i}(s)\right)\right. \\
& \left.+(-1)^{n} r(s)+f\left(s, x\left(\beta_{1}(s)\right), \ldots, x\left(\beta_{l}(s)\right)\right)\right) d s \\
= & 2 L+\frac{1}{(n-2) !} \int_{t}^{+\infty} d s \int_{t}^{s}(s-u)^{n-2}\left(\sum_{i=1}^{m} q_{i}(s) x\left(\alpha_{i}(s)\right)\right. \\
& \left.+(-1)^{n} r(s)+f\left(s, x\left(\beta_{1}(s)\right), \ldots, x\left(\beta_{l}(s)\right)\right)\right) d u \\
= & 2 L+\frac{1}{(n-1) !} \int_{t}^{+\infty}(s-t)^{n-1}\left(\sum_{i=1}^{m} q_{i}(s) x\left(\alpha_{i}(s)\right)\right. \\
& \left.+(-1)^{n} r(s)+f\left(s, x\left(\beta_{1}(s)\right), \ldots, x\left(\beta_{l}(s)\right)\right)\right) d s, \quad t \geq T+\tau,
\end{aligned}
$$


which yields

$$
\begin{aligned}
& \frac{d^{n}}{d t^{n}}[x(t)+x(t-\tau)]+(-1)^{n+1} \sum_{i=1}^{m} q_{i}(t) x\left(\alpha_{i}(t)\right) \\
& \quad+(-1)^{n+1} f\left(t, x\left(\beta_{1}(t)\right), \ldots, x\left(\beta_{l}(t)\right)\right)=r(t), \quad t \geq T+\tau,
\end{aligned}
$$

which together with (2.34) means that $x$ is a bounded nonoscillatory solution of (1.12) in $A(N, M)$.

Let $L_{1}, L_{2} \in(N, M)$ with $L_{1} \neq L_{2}$. For each $k \in\{1,2\}$, there exist a constant $T_{k}>\left|t_{0}\right|+\tau+$ $|c|+|\gamma|+n$ and a mapping $S_{L_{k}}: A(N, M) \rightarrow C B([\gamma,+\infty), \mathbb{R})$ satisfying (2.44) and (2.45), where $T, L$, and $S_{L}$ are replaced by $T_{k}, L_{k}$, and $S_{L_{k}}$, respectively, and (1.12) possesses a bounded nonoscillatory solution $z_{k} \in A(N, M)$. By (2.2), we choose some $T_{3}>\max \left\{T_{1}, T_{2}\right\}$ with

$$
\frac{1}{(n-1) !} \int_{T_{3}}^{+\infty} s^{n-1}\left(M \sum_{i=1}^{m}\left|q_{i}(s)\right|+h(s)\right) d s<\frac{\left|L_{1}-L_{2}\right|}{4} .
$$

Using (2.1), (2.45), and (2.51), we infer that for $t \geq T_{3}+\tau$

$$
\begin{aligned}
& \left|z_{1}(t)-z_{2}(t)\right| \\
& \geq\left|L_{1}-L_{2}\right|-\frac{1}{(n-2) !} \sum_{a=1}^{\infty} \int_{t+(2 a-1) \tau}^{t+2 a \tau} d u \int_{u}^{+\infty}(s-u)^{n-2} \\
& \times\left(\sum_{i=1}^{m}\left|q_{i}(s)\right|\left|z_{1}\left(\alpha_{i}(s)\right)-z_{2}\left(\alpha_{i}(s)\right)\right|\right. \\
& \left.+\left|f\left(s, z_{1}\left(\beta_{1}(s)\right), \ldots, z_{1}\left(\beta_{l}(s)\right)\right)-f\left(s, z_{2}\left(\beta_{1}(s)\right), \ldots, z_{2}\left(\beta_{l}(s)\right)\right)\right|\right) d s \\
& \geq\left|L_{1}-L_{2}\right|-\frac{2}{(n-2) !} \int_{t}^{+\infty} d u \int_{u}^{+\infty}(s-u)^{n-2}\left(M \sum_{i=1}^{m}\left|q_{i}(s)\right|+h(s)\right) d s \\
& =\left|L_{1}-L_{2}\right|-\frac{2}{(n-2) !} \int_{t}^{+\infty} d s \int_{t}^{s}(s-u)^{n-2}\left(M \sum_{i=1}^{m}\left|q_{i}(s)\right|+h(s)\right) d u \\
& =\left|L_{1}-L_{2}\right|-\frac{2}{(n-1) !} \int_{t}^{+\infty}(s-t)^{n-1}\left(M \sum_{i=1}^{m}\left|q_{i}(s)\right|+h(s)\right) d s \\
& >\left|L_{1}-L_{2}\right|-\frac{2\left|L_{1}-L_{2}\right|}{4} \\
& =\frac{\left|L_{1}-L_{2}\right|}{2}>0 \text {, }
\end{aligned}
$$

that is, $z_{1} \neq z_{2}$. Consequently (1.12) has uncountably bounded nonoscillatory solutions in $A(N, M)$. This completes the proof.

Theorem 2.7 Assume that there exist $h \in C\left(\left[t_{0},+\infty\right), \mathbb{R}^{+}\right)$and constants $M, N$, and c satisfying (2.1),

$$
\int_{t_{0}}^{+\infty} s^{n} \max \left\{\sum_{i=1}^{m}\left|q_{i}(s)\right|,|r(s)|, h(s)\right\} d s<+\infty
$$


and

$$
0<N<M, \quad p(t) \equiv-1, \quad \forall t \geq c \geq t_{0} .
$$

Then (1.12) has uncountably many bounded nonoscillatory solutions in $A(N, M)$.

Proof Let $L \in(N, M)$. It follows from (2.52) that there exists a constant $T>\tau+\left|t_{0}\right|+|c|+$ $|\gamma|+\frac{\left|t_{0}\right|}{\tau}+n$ satisfying

$$
\frac{1}{\tau(n-1) !} \int_{T}^{+\infty} s^{n} H(s) d s \leq \min \{M-L, L-N\}
$$

Let $t \geq t_{0}$ and $\left[\frac{s-t-T \tau}{\tau}\right]$ denote the largest integer not exceeding $\frac{s-t-T \tau}{\tau}$. Note that

$$
1+\left[\frac{s-t-T \tau}{\tau}\right] \leq 1+\frac{s-t-T \tau}{\tau} \leq \frac{s}{\tau}, \quad \forall s \geq t+T \tau
$$

and (2.52) implies that

$$
\begin{aligned}
\sum_{a=T}^{\infty} \int_{t+a \tau}^{+\infty}(s-t-a \tau)^{n-1} H(s) d s & \leq \sum_{a=T}^{\infty} \int_{t+a \tau}^{+\infty} s^{n-1} H(s) d s \\
& =\int_{t+T \tau}^{+\infty}\left(1+\left[\frac{s-t-T \tau}{\tau}\right]\right) s^{n-1} H(s) d s \\
& \leq \frac{1}{\tau} \int_{t+T \tau}^{+\infty} s^{n} H(s) d s<+\infty,
\end{aligned}
$$

which yields

$$
\sum_{a=1}^{\infty} \int_{t+a \tau}^{+\infty}(s-t-a \tau)^{n-1} H(s) d s<+\infty
$$

Define a mapping $S_{L}: A(N, M) \rightarrow C B\left(\left[t_{0},+\infty\right), \mathbb{R}\right)$ by

$$
\left(S_{L} x\right)(t)=\left\{\begin{array}{rlrl}
L+ & \frac{1}{(n-1) !} \sum_{a=1}^{\infty} \int_{t+a \tau}^{+\infty}(s-t-a \tau)^{n-1} & & \\
& \times\left(\sum_{i=1}^{m} q_{i}(s) x\left(\alpha_{i}(s)\right)+(-1)^{n} r(s)\right. & \\
& \left.+f\left(s, x\left(\beta_{1}(s)\right), \ldots, x\left(\beta_{l}(s)\right)\right)\right) d s, & & t \geq T, \\
\left(S_{L} x\right)(T), & & \gamma \leq t<T .
\end{array}\right.
$$

Notice that (2.1) and (2.55) mean that the mapping $S_{L}$ is well defined. Let $x \in A(N, M)$. In view of (2.1), (2.54), and (2.56), we conclude that for any $t \geq T$

$$
\begin{aligned}
\left|\left(S_{L} x\right)(t)-L\right|= & \frac{1}{(n-1) !} \mid \sum_{a=1}^{\infty} \int_{t+a \tau}^{+\infty}(s-t-a \tau)^{n-1}\left(\sum_{i=1}^{m} q_{i}(s) x\left(\alpha_{i}(s)\right)+(-1)^{n} r(s)\right. \\
& \left.+f\left(s, x\left(\beta_{1}(s)\right), \ldots, x\left(\beta_{l}(s)\right)\right)\right) d s \mid
\end{aligned}
$$




$$
\begin{aligned}
& \leq \frac{1}{(n-1) !} \sum_{a=1}^{\infty} \int_{t+a \tau}^{+\infty} s^{n-1} H(s) d s \\
& \leq \frac{1}{\tau(n-1) !} \int_{T}^{+\infty} s^{n} H(s) d s \\
& \leq \min \{M-L, L-N\},
\end{aligned}
$$

which shows that $S_{L}(A(N, M)) \subseteq A(N, M)$ and $S_{L}(A(N, M))$ is uniformly bounded in $[\gamma,+\infty)$.

Let $\left\{x_{k}\right\}_{k \in \mathbb{N}}$ be a sequence in $A(N, M)$ and $x \in A(N, M)$ satisfying (2.8) and let $G_{k}$ be defined by (2.37). By means of (2.37), (2.56), the continuity of $f, q_{i}, \alpha_{i}$ and $\beta_{j}$ for $i \in$ $\{1,2, \ldots, m\}$ and $j \in\{1,2, \ldots, l\}$, and the Lebesgue dominated convergence theorem, we deduce that for any $t \geq T$

$$
\begin{aligned}
& \left|\left(S_{L} x_{k}\right)(t)-\left(S_{L} x\right)(t)\right| \\
& \leq \frac{1}{(n-1) !} \sum_{a=1}^{\infty} \int_{t+a \tau}^{+\infty}(s-t-a \tau)^{n-1}\left(\sum_{i=1}^{m}\left|q_{i}(s)\right|\left|x_{k}\left(\alpha_{i}(s)\right)-x\left(\alpha_{i}(s)\right)\right|\right. \\
& \left.\quad+\left|f\left(s, x_{k}\left(\beta_{1}(t)\right), \ldots, x_{k}\left(\beta_{l}(t)\right)\right)-f\left(s, x\left(\beta_{1}(t)\right), \ldots, x\left(\beta_{l}(s)\right)\right)\right|\right) d s \\
& =\frac{1}{(n-1) !} \sum_{a=1}^{\infty} \int_{t+a \tau}^{+\infty} s^{n-1} G_{k}(s) d s \\
& \leq \frac{1}{\tau(n-1) !} \int_{T}^{+\infty} s^{n} G_{k}(s) d s \\
& \rightarrow 0 \quad \text { as } k \rightarrow \infty,
\end{aligned}
$$

which gives

$$
\lim _{k \rightarrow \infty}\left\|S_{L} x_{k}-S_{L} x\right\|=\lim _{k \rightarrow \infty} \sup _{t \geq T}\left|\left(S_{L} x_{k}\right)(t)-\left(S_{L} x\right)(t)\right|=0,
$$

that is, $S_{L}$ is continuous in $A(N, M)$.

Next we prove that $S_{L}(A(N, M))$ is equicontinuous in $[\gamma,+\infty)$. Given a positive number $\varepsilon$. It follows from (2.52) that there exists $T^{*}>T$ large enough satisfying

$$
\frac{1}{\tau(n-1) !} \int_{T^{*}}^{+\infty} s^{n} H(s) d s<\frac{\varepsilon}{8}
$$

Let $B$ and $K$ be defined by (2.39) and (2.40), respectively. Put

$$
\delta=\min \left\{1, \frac{\varepsilon}{8 K B}, \frac{\varepsilon}{4 K \tau(n-1) \min \{M-L, L-N\}}\right\} .
$$

Now we have to consider the following possible cases:

(i) $t_{2}>t_{1} \geq T^{*}$ with $\left|t_{1}-t_{2}\right|<\delta$. In view of (2.1) and (2.56)-(2.58), we get

$$
\begin{aligned}
& \left|\left(S_{L} x\right)\left(t_{1}\right)-\left(S_{L} x\right)\left(t_{2}\right)\right| \\
& \quad \leq \frac{1}{(n-1) !} \sum_{a=1}^{\infty}\left[\int _ { t _ { 1 } + a \tau } ^ { + \infty } ( s - t _ { 1 } - a \tau ) ^ { n - 1 } \left(\sum_{i=1}^{m}\left|q_{i}(s)\right|\left|x\left(\alpha_{i}(s)\right)\right|+|r(s)|\right.\right.
\end{aligned}
$$




$$
\begin{aligned}
& \left.\quad+\left|f\left(s, x\left(\beta_{1}(s)\right), \ldots, x\left(\beta_{l}(s)\right)\right)\right|\right) d s \\
& +\int_{t_{2}+a \tau}^{+\infty}\left(s-t_{2}-a \tau\right)^{n-1}\left(\sum_{i=1}^{m}\left|q_{i}(s)\right|\left|x\left(\alpha_{i}(s)\right)\right|+|r(s)|\right. \\
& \left.\left.\quad+\left|f\left(s, x\left(\beta_{1}(s)\right), \ldots, x\left(\beta_{l}(s)\right)\right)\right|\right) d s\right] \\
& \leq \frac{1}{(n-1) !} \sum_{a=1}^{\infty}\left(\int_{t_{1}+a \tau}^{+\infty} s^{n-1} H(s) d s+\int_{t_{2}+a \tau}^{+\infty} s^{n-1} H(s) d s\right) \\
& \leq \frac{1}{\tau(n-1) !}\left(\int_{t_{1}+\tau}^{+\infty} s^{n} H(s) d s+\int_{t_{2}+\tau}^{+\infty} s^{n} H(s) d s\right) \\
& \leq \frac{2}{\tau(n-1) !} \int_{T^{*}}^{+\infty} H(s) d s \\
& <\frac{\varepsilon}{4}, \quad \forall x \in A(N, M) .
\end{aligned}
$$

(ii) $T \leq t_{1}<t_{2} \leq T^{*}$ with $\left|t_{1}-t_{2}\right|<\delta$. Let $n \geq 2$. In light of (2.1), (2.54), (2.56)-(2.58), and the mean value theorem, we conclude that

$$
\begin{aligned}
& \left|\left(S_{L} x\right)\left(t_{1}\right)-\left(S_{L} x\right)\left(t_{2}\right)\right| \\
& \leq \frac{1}{(n-1) !} \sum_{a=1}^{2 K} \mid \int_{t_{1}+a \tau}^{+\infty}\left(s-t_{1}-a \tau\right)^{n-1}\left(\sum_{i=1}^{m} q_{i}(s) x\left(\alpha_{i}(s)\right)+(-1)^{n} r(s)\right. \\
& \left.+f\left(s, x\left(\beta_{1}(s)\right), \ldots, x\left(\beta_{l}(s)\right)\right)\right) d s \\
& -\int_{t_{1}+a \tau}^{+\infty}\left(s-t_{2}-a \tau\right)^{n-1}\left(\sum_{i=1}^{m} q_{i}(s) x\left(\alpha_{i}(s)\right)+(-1)^{n} r(s)\right. \\
& \left.+f\left(s, x\left(\beta_{1}(s)\right), \ldots, x\left(\beta_{l}(s)\right)\right)\right) d s \\
& +\int_{t_{1}+a \tau}^{+\infty}\left(s-t_{2}-a \tau\right)^{n-1}\left(\sum_{i=1}^{m} q_{i}(s) x\left(\alpha_{i}(s)\right)+(-1)^{n} r(s)\right. \\
& \left.+f\left(s, x\left(\beta_{1}(s)\right), \ldots, x\left(\beta_{l}(s)\right)\right)\right) d s \\
& -\int_{t_{2}+a \tau}^{+\infty}\left(s-t_{2}-a \tau\right)^{n-1}\left(\sum_{i=1}^{m} q_{i}(s) x\left(\alpha_{i}(s)\right)+(-1)^{n} r(s)\right. \\
& \left.+f\left(s, x\left(\beta_{1}(s)\right), \ldots, x\left(\beta_{l}(s)\right)\right)\right) d s \mid \\
& +\frac{1}{(n-1) !} \sum_{a=2 K+1}^{\infty}\left[\int _ { t _ { 1 } + a \tau } ^ { + \infty } ( s - t _ { 1 } - a \tau ) ^ { n - 1 } \left(\sum_{i=1}^{m}\left|q_{i}(s)\right|\left|x\left(\alpha_{i}(s)\right)\right|+|r(s)|\right.\right. \\
& \left.+\left|f\left(s, x\left(\beta_{1}(s)\right), \ldots, x\left(\beta_{l}(s)\right)\right)\right|\right) d s+\int_{t_{2}+a \tau}^{+\infty}\left(s-t_{2}-a \tau\right)^{n-1}
\end{aligned}
$$




$$
\begin{aligned}
&\left.\times\left(\sum_{i=1}^{m}\left|q_{i}(s)\right|\left|x\left(\alpha_{i}(s)\right)\right|+|r(s)|+\left|f\left(s, x\left(\beta_{1}(s)\right), \ldots, x\left(\beta_{l}(s)\right)\right)\right|\right) d s\right] \\
& \leq \frac{1}{(n-1) !} \sum_{a=1}^{2 K}\left[\int_{t_{1}+a \tau}^{+\infty}\left[\left(s-t_{1}-a \tau\right)^{n-1}-\left(s-t_{2}-a \tau\right)^{n-1}\right] H(s) d s\right. \\
&\left.+\int_{t_{1}+a \tau}^{t_{2}+a \tau}\left(t_{2}+a \tau-s\right)^{n-1} H(s) d s\right] \\
&+\frac{1}{(n-1) !} \sum_{a=2 K+1}^{\infty}\left(\int_{t_{1}+a \tau}^{+\infty} s^{n-1} H(s) d s+\int_{t_{2}+a \tau}^{+\infty} s^{n-1} H(s) d s\right) \\
& \leq \frac{1}{(n-1) !} \sum_{a=1}^{2 K}\left((n-1)\left|t_{2}-t_{1}\right| \int_{t_{1}+a \tau}^{+\infty} s^{n-2} H(s) d s+B\left|t_{2}-t_{1}\right|^{n}\right) \\
&+\frac{1}{\tau(n-1) !}\left(\int_{t_{1}+(2 K+1) \tau}^{+\infty} s^{n} H(s) d s+\int_{t_{2}+(2 K+1) \tau}^{+\infty} s^{n} H(s) d s\right) \\
& \leq \frac{2 K \delta}{(n-1) !}\left((n-1) \int_{T}^{+\infty} s^{n-1} H(s) d s+B\right) \\
&+\frac{2}{\tau(n-1) !} \int_{T^{*}}^{+\infty} s^{n} H(s) d s \\
&< \frac{\varepsilon}{2}+\frac{\varepsilon}{4}+\frac{\varepsilon}{4} \\
&<\varepsilon
\end{aligned}
$$

Let $n=1$. By means of (2.1), (2.54), and (2.56)-(2.58), we get

$$
\begin{aligned}
\mid & \left(S_{L} x\right)\left(t_{1}\right)-\left(S_{L} x\right)\left(t_{2}\right) \mid \\
= & \mid \sum_{a=1}^{\infty} \int_{t_{1}+a \tau}^{+\infty}\left(\sum_{i=1}^{m} q_{i}(s) x\left(\alpha_{i}(s)\right)-r(s)+f\left(s, x\left(\beta_{1}(s)\right), \ldots, x\left(\beta_{l}(s)\right)\right)\right) d s \\
& \quad-\sum_{a=1}^{\infty} \int_{t_{2}+a \tau}^{+\infty}\left(\sum_{i=1}^{m} q_{i}(s) x\left(\alpha_{i}(s)\right)-r(s)+f\left(s, x\left(\beta_{1}(s)\right), \ldots, x\left(\beta_{l}(s)\right)\right)\right) d s \mid \\
& \leq \sum_{a=1}^{2 K} \int_{t_{1}+a \tau}^{t_{2}+a \tau} H(s) d s+\sum_{a=2 K+1}^{\infty}\left(\int_{t_{1}+a \tau}^{+\infty} H(s) d s+\int_{t_{2}+a \tau}^{+\infty} H(s) d s\right) \\
& \leq 2 K B\left|t_{2}-t_{1}\right|+\frac{2}{\tau} \int_{T^{*}}^{+\infty} s H(s) d s \\
& <\frac{\varepsilon}{4}+\frac{\varepsilon}{4} \\
& <\varepsilon .
\end{aligned}
$$

(iii) $\gamma \leq t_{1}<t_{2} \leq T$ with $\left|t_{1}-t_{2}\right|<\delta$. It is easy to see that

$$
\left|\left(S_{L} x\right)\left(t_{2}\right)-\left(S_{L} x\right)\left(t_{1}\right)\right|=0<\varepsilon .
$$

It is easy to see that (2.59)-(2.62) ensure that $S_{L}(A(N, M))$ is equicontinuous in $[\gamma,+\infty)$. Consequently $S_{L}(A(N, M))$ is relatively compact. Thus Lemma 1.2 implies that $S_{L}$ pos- 
sesses a fixed point $x \in A(N, M)$, that is,

$$
\begin{aligned}
x(t)= & L+\frac{1}{(n-1) !} \sum_{a=1}^{\infty} \int_{t+a \tau}^{+\infty}(s-t-a \tau)^{n-1}\left(\sum_{i=1}^{m} q_{i}(s) x\left(\alpha_{i}(s)\right)+(-1)^{n} r(s)\right. \\
& \left.+f\left(s, x\left(\beta_{1}(s)\right), \ldots, x\left(\beta_{l}(s)\right)\right)\right) d s, \quad t \geq T+\tau
\end{aligned}
$$

and

$$
\begin{aligned}
x(t-\tau)= & L+\frac{1}{(n-1) !} \sum_{a=1}^{\infty} \int_{t+(a-1) \tau}^{+\infty}(s-t-(a-1) \tau)^{n-1}\left(\sum_{i=1}^{m} q_{i}(s) x\left(\alpha_{i}(s)\right)+(-1)^{n} r(s)\right. \\
& \left.+f\left(s, x\left(\beta_{1}(s)\right), \ldots, x\left(\beta_{l}(s)\right)\right)\right) d s, \quad t \geq T+\tau,
\end{aligned}
$$

which imply that

$$
\begin{aligned}
& \frac{d^{n}}{d t^{n}}[x(t)-x(t-\tau)]+(-1)^{n+1} \sum_{i=1}^{m} q_{i}(t) x\left(\alpha_{i}(t)\right) \\
& \quad+(-1)^{n+1} f\left(t, x\left(\beta_{1}(t)\right), \ldots, x\left(\beta_{l}(t)\right)\right)=r(t), \quad t \geq T+\tau,
\end{aligned}
$$

which together with (2.53) means that $x$ is a bounded nonoscillatory solution of (1.12) in $A(N, M)$.

Let $L_{1}$ and $L_{2}$ be two different numbers in $(N, M)$. Similar to the above proof, we infer that there exist constants $T_{1}, T_{2}$ and mappings $S_{L_{1}}, S_{L_{2}}: A(N, M) \rightarrow C B\left(\left[t_{0},+\infty\right), \mathbb{R}\right)$ defined by (2.56), where $L, T$, and $S_{L}$ are replaced by $L_{k}, T_{k}$, and $S_{L_{k}}$, respectively, and $T_{k}>\tau+\left|t_{0}\right|+|c|+|\gamma|+\frac{\left|t_{0}\right|}{\tau}+n$ for $k \in\{1,2\}$, such that $S_{L_{1}}$ and $S_{L_{2}}$ have, respectively, fixed points $z_{1}$ and $z_{2} \in A(N, M)$, which are bounded nonoscillatory solutions of (1.12) in $A(N, M)$, that is,

$$
\begin{aligned}
z_{k}(t)= & L_{k}+\frac{1}{(n-1) !} \sum_{a=1}^{\infty} \int_{t+a \tau}^{+\infty}(s-t-a \tau)^{n-1}\left(\sum_{i=1}^{m} q_{i}(s) z_{k}\left(\alpha_{i}(s)\right)+(-1)^{n} r(s)\right. \\
& \left.+f\left(s, z_{k}\left(\beta_{1}(s)\right), \ldots, z_{k}\left(\beta_{l}(s)\right)\right)\right) d s, \quad t \geq T_{k}
\end{aligned}
$$

Note that (2.52) ensures that there exists $T_{3}>\max \left\{T_{1}, T_{2}\right\}$ satisfying

$$
\frac{1}{\tau(n-1) !} \int_{T_{3}}^{+\infty} s^{n} \max \left\{\sum_{i=1}^{m}\left|q_{i}(s)\right|, h(s)\right\} d s<\min \left\{1, \frac{\left|L_{1}-L_{2}\right|}{4}\right\} .
$$

Combining (2.1), (2.63), and (2.64), we deduce that for any $t \geq T_{3}$

$$
\begin{aligned}
& \left|z_{1}(t)-z_{2}(t)\right| \\
& \quad \geq\left|L_{1}-L_{2}\right|-\frac{1}{(n-1) !} \sum_{a=1}^{\infty}\left[\int _ { t + a \tau } ^ { + \infty } ( s - t - a \tau ) ^ { n - 1 } \left(\sum_{i=1}^{m}\left|q_{i}(s)\right|\left|z_{1}\left(\alpha_{i}(s)\right)-z_{2}\left(\alpha_{i}(s)\right)\right|\right.\right.
\end{aligned}
$$




$$
\begin{aligned}
& \left.\left.+\left|f\left(s, z_{1}\left(\beta_{1}(s)\right), \ldots, z_{1}\left(\beta_{l}(s)\right)\right)-f\left(s, z_{2}\left(\beta_{1}(s)\right), \ldots, z_{2}\left(\beta_{l}(s)\right)\right)\right|\right) d s\right] \\
\geq & \left|L_{1}-L_{2}\right|-\frac{1}{(n-1) !} \sum_{a=1}^{\infty} \int_{t+a \tau}^{+\infty} s^{n-1}\left(\sum_{i=1}^{m}\left|q_{i}(s)\right|\left\|z_{1}-z_{2}\right\|+2 h(s)\right) d s \\
\geq & \left|L_{1}-L_{2}\right|-\frac{1}{\tau(n-1) !} \int_{t+\tau}^{+\infty} s^{n}\left(\sum_{i=1}^{m}\left|q_{i}(s)\right|\left\|z_{1}-z_{2}\right\|+2 h(s)\right) d s \\
\geq & \left|L_{1}-L_{2}\right|-\frac{\left\|z_{1}-z_{2}\right\|}{\tau(n-1) !} \int_{T_{3}}^{+\infty} s^{n} \sum_{i=1}^{m}\left|q_{i}(s)\right| d s-\frac{2}{\tau(n-1) !} \int_{T_{3}}^{+\infty} s^{n} h(s) d s \\
> & \left|L_{1}-L_{2}\right|-\left\|z_{1}-z_{2}\right\|-\frac{2\left|L_{1}-L_{2}\right|}{4} \\
= & \frac{\left|L_{1}-L_{2}\right|}{2}-\left\|z_{1}-z_{2}\right\|,
\end{aligned}
$$

which implies that

$$
\left\|z_{1}-z_{2}\right\|>\frac{\left|L_{1}-L_{2}\right|}{4}>0
$$

that is, $z_{1} \neq z_{2}$. Consequently, (1.12) has uncountably bounded nonoscillatory solutions in $A(N, M)$. This completes the proof.

Remark 2.1 Theorems 2.1-2.7 extend, improve, and unify the theorem in [4], Theorems 2 and 3 in [13], Theorems 1-3 in [14], Theorems 1-6 in [15], and Theorems 1-4 in [16].

\section{Examples and applications}

Now we construct six nontrivial examples to show the superiority and applications of the results presented in the second section.

Example 3.1 Consider the higher-order neutral delay differential equation:

$$
\begin{aligned}
& \frac{d^{n}}{d t^{n}}\left[x(t)+\frac{1-t^{2}}{1+3 t^{2}} x(t-\tau)\right]+(-1)^{n+1}\left[\frac{\ln \left(1+t^{2}\right)}{1+t^{n+2}} x\left(t^{2}\right)-\frac{1-3 t^{2}}{1+t^{n+3}} x(\sqrt{t+1})\right] \\
& +\frac{(-1)^{n+1} t^{7} x^{5}(2 t)+(-1)^{n}\left(t^{4}+2\right) x^{2}\left(t^{3}\right)}{1+t^{n+8}+t^{2} x^{2}(3 t-1)}=\frac{1-100 t+t^{3}}{1+t^{n+4}}, \quad t \geq 0,
\end{aligned}
$$

where $\tau>0$ is a constant. Let $t_{0}=0, c=1, m=2, l=3, M=20, N=9, p_{0}=-\frac{1}{2}$,

$$
\begin{aligned}
& p(t)=\frac{1-t^{2}}{1+3 t^{2}}, \quad \alpha_{1}(t)=t^{2}, \quad \alpha_{2}(t)=\sqrt{t+1}, \\
& \beta_{1}(t)=2 t, \quad \beta_{2}(t)=t^{3}, \quad \beta_{3}(t)=3 t-1, \quad r(t)=\frac{1-100 t+t^{3}}{1+t^{n+4}}, \\
& q_{1}(t)=\frac{\ln \left(1+t^{2}\right)}{1+t^{n+2}}, \quad q_{2}(t)=-\frac{1-3 t^{2}}{1+t^{n+3}}, \quad h(t)=\frac{M^{2}\left(M^{3} t^{7}+t^{4}+2\right)}{1+t^{n+8}+t^{2} N^{2}}, \\
& f(t, u, v, w)=\frac{t^{7} u^{5}-\left(t^{4}+2\right) v^{2}}{1+t^{n+8}+t^{2} w^{2}}, \quad \forall(t, u, v, w) \in\left[t_{0},+\infty\right) \times \mathbb{R}^{3} .
\end{aligned}
$$


It is easy to verify that (2.1)-(2.3) are fulfilled. Thus Theorem 2.1 ensures that (3.1) has uncountably many bounded nonoscillatory solutions in $A(N, M)$. But the theorem in [4], Theorems 2 and 3 in [13], Theorems 1-3 in [14], Theorems 1-6 in [15], and Theorems 1-4 in [16] are invalid for (3.1).

Example 3.2 Consider the higher-order neutral delay differential equation:

$$
\begin{aligned}
& \frac{d^{n}}{d t^{n}}\left[x(t)+\frac{3 t^{2}}{1+4 t^{2}} x(t-\tau)\right]+(-1)^{n+1}\left[\frac{1-2 t^{2}}{1+t^{n+4}} x\left(t^{3}\right)+\frac{(1-t) \ln \left(1+t^{2}\right)}{1+t^{n+2}} x\left(t^{2}\right)\right] \\
& \quad+(-1)^{n+1}\left[\frac{1-2 t^{10} x^{2}(t-1) x^{5}(3 t-2)-t^{3} \ln \left(1+t^{2} x^{2}(2 t-1)\right)}{1+2 t^{n+11}+t\left|(1-t) x\left(t^{3}-2 t+1\right)\right|}\right] \\
& =\frac{\sqrt{t}-t^{3}}{1+t^{2 n+4}}, \quad t \geq 0,
\end{aligned}
$$

where $\tau>0$ is a constant, $t_{0}=c=0, m=2, l=4, M=24, N=5, p_{0}=\frac{3}{4}$,

$$
\begin{aligned}
& p(t)=\frac{3 t^{2}}{1+4 t^{2}}, \quad \alpha_{1}(t)=t^{3}, \quad \alpha_{2}(t)=t^{2}, \quad \beta_{1}(t)=t-1, \\
& \beta_{2}(t)=3 t-2, \quad \beta_{3}(t)=2 t-1, \quad \beta_{4}(t)=t^{3}-2 t+1, \quad r(t)=\frac{\sqrt{t}-t^{3}}{1+t^{2 n+4}}, \\
& q_{1}(t)=\frac{1-2 t^{2}}{1+t^{n+4}}, \quad q_{2}(t)=\frac{(1-t) \ln \left(1+t^{2}\right)}{1+t^{n+2}}, \quad h(t)=\frac{1+2 M^{7} t^{10}+t^{3} \ln \left(1+t^{2} M^{2}\right)}{1+2 t^{n+11}+N t|1-t|}, \\
& f(t, u, v, w, z)=\frac{1-2 t^{10} u^{2} v^{5}-t^{3} \ln \left(1+t^{2} z^{2}\right)}{1+2 t^{n+11}+t|(1-t) w|}, \quad \forall(t, u, v, w, z) \in\left[t_{0},+\infty\right) \times \mathbb{R}^{4} .
\end{aligned}
$$

Obviously, (2.1), (2.2), and (2.18) hold. It follows from Theorem 2.2 that (3.2) has uncountably many bounded nonoscillatory solutions in $A(N, M)$. However, the theorem in [4], Theorems 2 and 3 in [13], Theorems 1-3 in [14], Theorems 1-6 in [15], and Theorems 1-4 in [16] are unapplicable for (3.2).

Example 3.3 Consider the higher-order neutral delay differential equation:

$$
\begin{aligned}
& \frac{d^{n}}{d t^{n}}\left[x(t)+\left(1+\frac{1}{t}\right)^{t} x(t-\tau)\right]+\frac{(3-t) x(t-\sqrt{t})}{\left(1+t^{2}\right)\left(t^{n}+\sqrt{1+\sin ^{2} t}\right)} \\
& \quad+\frac{(1+t) x^{5}\left(t^{2}-\ln \left(1+t^{2}\right)\right)}{1+t^{2} x^{2}\left(t^{2}-\ln \left(1+t^{2}\right)\right)+t^{n+2}}=\frac{1-(t+1)^{n}+t^{2 n}}{\sqrt{1+\cos ^{2}\left(2 t^{2}-1\right)}+t^{3 n+2}}, \quad t \geq 1,
\end{aligned}
$$

where $\tau>0$ is a constant. Let $t_{0}=c=m=l=1, M=400, N=50, p_{0}=2, p_{1}=3$,

$$
\begin{aligned}
& p(t)=\left(1+\frac{1}{t}\right)^{t}, \quad \alpha_{1}(t)=t-\sqrt{t}, \quad \beta_{1}(t)=t^{2}-\ln \left(1+t^{2}\right), \\
& r(t)=\frac{1-(t+1)^{n}+t^{2 n}}{\sqrt{1+\cos ^{2}\left(2 t^{2}-1\right)}+t^{3 n+2}}, \quad q_{1}(t)=\frac{(-1)^{n+1}(3-t)}{\left(1+t^{2}\right)\left(t^{n}+\sqrt{1+\sin ^{2} t}\right)}, \\
& h(t)=\frac{M^{5}(1+t)}{1+N^{2} t^{2}+t^{n+2}}, \quad f(t, u)=\frac{(-1)^{n+1}(1+t) u^{5}}{1+t^{2} u^{2}+t^{n+2}}, \quad \forall(t, u) \in\left[t_{0},+\infty\right) \times \mathbb{R} .
\end{aligned}
$$

It is easy to verify that (2.1), (2.2), and (2.20) are fulfilled. Consequently Theorem 2.3 means that (3.3) has uncountably many bounded nonoscillatory solutions in $A(N, M)$. But the 
theorem in [4], Theorems 2 and 3 in [13], Theorems 1-3 in [14], Theorems 1-6 in [15], and Theorems 1-4 in [16] are not applicable for (3.3).

Example 3.4 Consider the higher-order neutral delay differential equation:

$$
\begin{aligned}
& \frac{d^{n}}{d t^{n}}\left[x(t)-\frac{4+3 t^{2}}{3+2 t^{2}} x(t-\tau)\right]+\frac{(-1)^{n+1}(1-2 t)}{t^{n}\left(1+t^{\frac{5}{3}}\right)} x\left(t^{2}-3 \sqrt{t^{2}+1}+9\right) \\
& \quad+\frac{\left(1-4 t^{2}\right) x^{9}\left(t^{3}-2\right)}{\left(1+t^{3}\right)\left(1+t^{n}\right)+t^{4} x^{2}\left(t^{3}-2\right)}=\frac{1-t^{3}+5 t^{12}}{t^{n+15}+\ln \left(1+\sqrt{1+t^{2}}\right)}, \quad t \geq 1,
\end{aligned}
$$

where $\tau>0$ is a constant. Let $t_{0}=1, c=2, m=l=1, M=33, N=27, p_{0}=-\frac{4}{3}, p_{1}=-\frac{3}{2}$,

$$
\begin{aligned}
& p(t)=-\frac{4+3 t^{2}}{3+2 t^{2}}, \quad \alpha_{1}(t)=t^{2}-3 \sqrt{t^{2}+1}+9, \\
& \beta_{1}(t)=t^{3}-2, \quad r(t)=\frac{1-t^{3}+5 t^{12}}{t^{n+15}+\ln \left(1+\sqrt{1+t^{2}}\right)}, \\
& q_{1}(t)=\frac{(-1)^{n+1}(1-2 t)}{t^{n}\left(1+t^{\frac{5}{3}}\right)}, \quad h(t)=\frac{\left(1+4 t^{2}\right) M^{9}}{\left(1+t^{3}\right)\left(1+t^{n}\right)+t^{4} N^{2}}, \\
& f(t, u)=\frac{(-1)^{n+1}\left(1-4 t^{2}\right) u^{9}}{\left(1+t^{3}\right)\left(1+t^{n}\right)+t^{4} u^{2}}, \quad \forall(t, u) \in\left[t_{0},+\infty\right) \times \mathbb{R} .
\end{aligned}
$$

Obviously, (2.1), (2.2), and (2.32) hold. Consequently Theorem 2.4 shows that (3.4) has uncountably many bounded nonoscillatory solutions in $A(N, M)$. But the theorem in [4], Theorems 2 and 3 in [13], Theorems 1-3 in [14], Theorems 1-6 in [15], and Theorems 1-4 in [16] are useless for (3.4).

Example 3.5 Consider the higher-order neutral delay differential equation:

$$
\begin{aligned}
& \frac{d^{n}}{d t^{n}}[x(t)+x(t-\tau)]+\frac{\left(t^{2}-\frac{1}{t}\right) \sin \sqrt{t-2}}{\left(1+t^{3}\right)\left(t^{n}+2\right)} x\left(3 t^{3}-24\right) \\
& \quad+\frac{\left(1+t^{3} \sin \left(1-t^{4}\right)\right) x^{5}(3 t-2)}{\left(1+t^{2}\right) t^{n+2}+t^{2} x^{2}(3 t-2)+t^{6} \sqrt{1+t} x^{4}(2 t)}=\frac{2-3 t^{4}}{t^{5}\left(2+t^{n}\right)}, \quad t \geq 2,
\end{aligned}
$$

where $\tau>0$ is a constant. Let $t_{0}=2, c=3, m=1, l=2, M=4, N=2$,

$$
\begin{aligned}
& p(t)=1, \quad \alpha_{1}(t)=3 t^{3}-24, \quad \beta_{1}(t)=3 t-2, \quad \beta_{2}(t)=2 t, \quad r(t)=\frac{2-3 t^{4}}{t^{5}\left(2+t^{n}\right)} \\
& q_{1}(t)=\frac{(-1)^{n+1}\left(t^{2}-\frac{1}{t}\right) \sin \sqrt{t-2}}{\left(1+t^{3}\right)\left(t^{n}+2\right)}, \quad h(t)=\frac{\left(1+t^{3}\right) M^{5}}{\left(1+t^{2}\right) t^{n+2}+t^{2} N^{2}+t^{6} \sqrt{1+t} N^{4}} \\
& f(t, u, v)=\frac{(-1)^{n+1}\left(1+t^{3} \sin \left(1-t^{4}\right)\right) u^{5}}{\left(1+t^{2}\right) t^{n+2}+t^{2} u^{2}+t^{6} \sqrt{1+t} v^{4}}, \quad \forall(t, u, v) \in\left[t_{0},+\infty\right) \times \mathbb{R}^{2} .
\end{aligned}
$$

It is clear that (2.1), (2.2), and (2.34) hold. Consequently Theorems 2.5 and 2.6 ensure that (3.5) has uncountably many bounded nonoscillatory solutions in $A(N, M)$. But the theorem in [4], Theorems 2 and 3 in [13], Theorems 1-3 in [14], Theorems 1-6 in [15], and Theorems 1-4 in [16] are null for (3.5). 
Example 3.6 Consider the higher-order neutral delay differential equation:

$$
\begin{gathered}
\frac{d^{n}}{d t^{n}}[x(t)-x(t-\tau)]+\frac{1-t-t^{5}}{\left(1+t^{5}\right)\left(1+t^{n+\frac{3}{2}}\right)} x\left(t^{2}-1\right) \\
+\frac{\left(t^{2}-\left(1+t^{3}\right) \sqrt{2 t-6}\right) \sqrt{1+t^{4} x^{2}(5 t-1)}}{\left(1+t^{7}\right)\left(1+t^{n-1}+t^{n} x^{2}(5 t-1)\right)} \\
=\frac{2-t^{3} \ln \left(1+\sqrt{1+2 t+5 t^{3}}\right)}{\left(1+t^{5}\right)\left(1+2 t^{2}+t^{n}\right)}, \quad t \geq 3,
\end{gathered}
$$

where $\tau>0$ is a constant. Let $t_{0}=3, c=3, m=l=1, M=6, N=5$,

$$
\begin{aligned}
& p(t)=-1, \quad \alpha_{1}(t)=t^{2}-1, \quad \beta_{1}(t)=5 t-1, \\
& r(t)=\frac{2-t^{3} \ln \left(1+\sqrt{1+2 t+5 t^{3}}\right)}{\left(1+t^{5}\right)\left(1+2 t^{2}+t^{n}\right)}, \quad q_{1}(t)=\frac{(-1)^{n+1}\left(1-t-t^{5}\right)}{\left(1+t^{5}\right)\left(1+t^{n+\frac{3}{2}}\right)}, \\
& h(t)=\frac{\left(t^{2}-\left(1+t^{3}\right) \sqrt{2 t-6}\right) \sqrt{1+t^{4} M^{2}}}{\left(1+t^{7}\right)\left(1+t^{n-1}+t^{n} N^{2}\right)}, \\
& f(t, u)=\frac{(-1)^{n+1}\left(t^{2}-\left(1+t^{3}\right) \sqrt{2 t-6}\right) \sqrt{1+t^{4} u^{2}}}{\left(1+t^{7}\right)\left(1+t^{n-1}+t^{n} u^{2}\right)}, \quad \forall(t, u) \in\left[t_{0},+\infty\right) \times \mathbb{R} .
\end{aligned}
$$

It is clear that (2.1), (2.2), and (2.52) hold. Consequently Theorem 2.7 shows that (3.6) has uncountably many bounded nonoscillatory solutions in $A(N, M)$. But the theorem in [4], Theorems 2 and 3 in [13], Theorems 1-3 in [14], Theorems 1-6 in [15], and Theorems 1-4 in [16] are void for (3.6).

\section{Competing interests}

The authors declare that they have no competing interests.

\section{Authors' contributions}

All the authors contributed equally and significantly in writing this paper. All authors read and approved the final manuscript.

\section{Author details}

${ }^{1}$ Department of Mathematics, Liaoning Normal University, Dalian, Liaoning 116029, People's Republic of China. ${ }^{2}$ Dalian Hongwen Middle School, Dalian, Liaoning 116029, People's Republic of China. ${ }^{3}$ Department of Mathematics, Changwon National University, Changwon, 641-773, Korea. ${ }^{4}$ Department of Mathematics, Research Institute of Natural Science, Gyeongsang National University, Jinju, 660-701, Korea.

\section{Acknowledgements}

This research was supported by the Science Research Foundation of Educational Department of Liaoning Province (L2012380) and Basic Science Research Program through the National Research Foundation of Korea (NRF) funded by the Ministry of Science, ICT \& Future Planning (2013R1A1A2057665).

\section{Received: 20 February 2014 Accepted: 8 July 2014 Published: 19 Aug 2014}

\section{References}

1. Erbe, LH, Kong, QK, Zhang, BG: Oscillation theory for functional differential equations. Dekker, New York (1995)

2. Györi, I, Ladas, G: Oscillation theory of delay differential equations with applications. Oxford University Press, London (1991)

3. Kong, QK, Sun, YJ, Zhang, BG: Nonoscillation of a class of neutral differential equations. Comput. Math. Appl. 44 643-654 (2002)

4. Kulenović, MRS, Hadžiomerspahić, S: Existence of nonoscillatory solution of second order linear neutral delay equation. J. Math. Anal. Appl. 228, 436-448 (1998)

5. Li, XP, Liu, YH, Zhou, TJ: The existence of positive solution and bounded oscillation for even-order neutral differential equations with unstable type. Appl. Math. Comput. 176, 632-641 (2006) 
6. Liu, BW, Huang, LH: Existence and uniqueness of periodic solutions for a kind of first order neutral functional differential equations with a deviating argument. Taiwan. J. Math. 11, 497-510 (1997)

7. Liu, Z, Gao, HY, Kang, SM, Shim, SH: Existence and Mann iterative approximations of nonoscillatory solutions of nth-order neutral delay differential equations. J. Math. Anal. Appl. 329, 515-529 (2007)

8. Liu, Z, Gao, HY, Shim, SH, Kang, SM: Existence and iterative approximations of bounded nonoscillatory solutions of higher-order neutral delay differential equations. Dyn. Contin. Discrete Impuls. Syst., Ser. A Math. Anal. 16, 701-723 (2009)

9. Luo, ZG, Shen, JH: Oscillation and nonoscillation of neutral differential equations with positive and negative coefficients. Czechoslov. Math. J. 54(129), 79-93 (2004)

10. Öcalan, Ö: Existence of positive solutions for a neutral differential equations with positive and negative coefficients. Appl. Math. Lett. 22, 84-90 (2009)

11. Parhi, N: Oscillations of higher order differential equations of neutral type. Czechoslov. Math. J. 50(125), 155-173 (2000)

12. Shen, JH, Debnath, L: Oscillations of solutions of neutral differential equations with positive and negative coefficients Appl. Math. Lett. 14, 775-781 (2001)

13. Yu, JS, Wang, ZC, Qian, C: Oscillation and nonoscillation of neutral differential equations. Bull. Aust. Math. Soc. 45, 195-200 (1992)

14. Zhang, WP, Fang, W, Yan, JR, Song, JS: Existence of nonoscillatory solutions of first-order linear neutral delay differential equations. Comput. Math. Appl. 49, 1021-1027 (2005)

15. Zhou, Y, Zhang, BG: Existence for nonoscillatory solutions of higher order of nonlinear neutral differential equations. Czechoslov. Math. J. 55(130), 237-253 (2005)

16. Zhou, Y, Zhang, BG: Existence of nonoscillatory solutions of higher-order neutral differential equations with positive and negative coefficients. Appl. Math. Lett. 15, 867-874 (2002)

10.1186/1029-242X-2014-302

Cite this article as: Liu et al.: Nonoscillatory solutions for higher-order nonlinear neutral delay differential equations. Journal of Inequalities and Applications 2014, 2014:302

\section{Submit your manuscript to a SpringerOpen ${ }^{0}$ journal and benefit from:}

- Convenient online submission

- Rigorous peer review

- Immediate publication on acceptance

- Open access: articles freely available online

- High visibility within the field

- Retaining the copyright to your article 